\title{
BUILDING COSMOLOGICAL MODELS VIA NONCOMMUTATIVE GEOMETRY
}

\author{
MATILDE MARCOLLI
}

\section{…万类霜天竞自由. 怅胗廓, 问苍范大地, 谁主沉浮 ? \\ (from "Changsha", 1925)}

\begin{abstract}
This is an overview of new and ongoing research developments aimed at constructing cosmological models based on noncommutative geometry, via the spectral action functional, thought of as a modified gravity action, which includes the coupling with matter when computed on an almost commutative geometry. This survey is mostly based on recent results obtained in collaboration with Elena Pierpaoli and Kevin Teh. We describe various aspects of cosmological models of the very early universe, developed by the author and Pierpaoli, based on the asymptotic expansion of the spectral action functional and on renormalization group analysis of the associated particle physics model (an extension of the standard model with right handed neutrinos and Majorana mass terms previously developed in collaboration with Chamseddine and Connes). We also describe non-perturbative results, more recently obtained by Pierpaoli, Teh, and the author, which extend to the more modern universe, which show that, for different candidate cosmic topologies, the form of the slow-roll inflation potentials obtained from the non-perturbative calculation of the spectral action are strongly coupled to the underlying geometry. We discuss some ongoing directions of research and open questions in this new field of "noncommutative cosmology". The paper is based on the talk given by the author at the conference "Geometry and Quantum Field Theory" at the MPI, in honor of Alan Carey.
\end{abstract}

Dedicated to Alan Carey, on the occasion of his $60^{\text {th }}$ birthday

\section{InTRODUCTION: MATHEMATICAL MODELS AND COSMOLOGICAL DATA}

Cosmology is currently undergoing one of the most exciting phases of rapid development, with sophisticated theoretical modeling being tested against very accurate observational data for both the Cosmic Microwave Background (CMB) and the matter distribution in the Universe. This is therefore a highly appropriate time for a broad range of mathematical models of particle physics and cosmology to formulate testable predictions that can be confronted with the data.

While model building within the framework of string and brane scenarios and their possible implications for particle physics and cosmology have been widely developed in recent years, less attention has been devoted to other sources of theoretical high energy physics models that are capable of producing a range of predictions, both in the particle physics and cosmology context. It is especially interesting to look for alternative models, which deliver predictions that are distinguishable from those obtained within the framework of string theory. Particle physics models derived within the framework of Noncommutative Geometry recently emerged as a source for new cosmological models, [11], [45], [46], [51], [52], [53], [54].

Among the most interesting features of these models of particle physics based on noncommutative geometry is the fact that the physical Lagrangian of the model is completely computed from a simple geometric input (the choice of a finite dimensional algebra), so that the physics is very tightly constrained by the underlying geometry.

The features that link the noncommutative geometry models to areas of current interest to theoretical cosmologists are the fact that the action functional of these models, the spectral action, behaves in the large energy asymptotic expansion like a modified gravity model, with additional coupling to 
matter. Various models of modified gravity have been extensively studied by theoretical cosmologists in recent years. Another feature, which is particular to the noncommutative geometry models we consider here, is the fact that the nonperturbative form of the spectral action determines a slow-roll inflation potential, which shows a coupling of spatial geometry (cosmic topology) and the possible inflation scenarios. The model also exhibits couplings of matter and gravity, which provide early universe models with variable effective gravitational and cosmological constants.

The results described in this paper are mostly based on recent joint work with Elena Pierpaoli [45], with Elena Pierpaoli and Kevin Teh [46], and with Daniel Kolodrubetz [38], as well as on earlier joint work with Ali Chamseddine and Alain Connes [21].

1.1. Noncommutative geometry methods. Within the framework of noncommutative geometry, a generalization of classical Riemannian geometry is provided by the notion of a spectral triple (a noncommutative Riemannian manifold), see [24]. A triple consists of a (non-commutative) algebra of coordinates, a Hilbert space representation, and an unbounded Dirac operator, from which the Riemannian metric can be recovered in the classical case. This notion allows one to apply techniques and results from smooth and metric geometry, suitably reformulated in spectral terms, to objects as varied as fractals, quantum groups, and various examples of noncommutative spaces with no classical analog. What is interesting from a physical perspective is that spectral triples come with an action functional, the spectral action introduced in [16], [17], expressed in terms of the Dirac operator of the spectral triple. The spectral action has an asymptotic expansion in terms of an energy scale and the coefficients of this expansion deliver interaction terms for a certain number of fermionic and bosonic fields which parameterize the representation of the algebra and the Dirac operator with its "inner fluctuations" induced by Morita equivalences of the algebra. Since the mid '90s, [25], noncommutative geometry has been proposed as a possible source of mathematical models for particle physics. The early models developed with this method concentrated on recovering the minimal standard model (MSM), while it was generally believed for several years that physics beyond the standard model, such as neutrino mixing and oscillations [49], would not be accessible to this approach. This all changed recently, when the results of [4], [26], [21] showed that the full Lagrangian of the standard model, coupled to gravity in an interesting way that will be discussed more extensively below, together with right handed neutrinos with Dirac and Majorana mass terms could be computed as the asymptotic expansion of a spectral action functional (see [16], [17]) on a noncommutative space that is a product of an ordinary spacetime manifold by a finite noncommutative geometry, that is, an almost commutative geometry.

Some particle physics implications of the model were discussed in [21]. In view of the cosmological applications we review in the following, an interesting property of the model lies in the presence, in the Lagrangian generated by the high energy expansion of the spectral action, of gravity and cosmological terms with interesting coupling to the particle physics content of the model, through an explicit dependence of the effective gravitational and cosmological constants of the model on the Yukawa parameters of the particle physics sector, which depends on the geometry of the Dirac operators on the noncommutative space.

\section{NONCOMMUTATIVE GEOMETRY FROM PARTICle PHySics TO COSMOLOGY}

This section gives an overview of results of my earlier collaboration with Chamseddine and Connes [21], which provides the background particle physics model, for which the cosmological applications are then further investigated.

The general approach followed in the construction of this type of models of high energy physics can be described broadly as a model with "extra dimensions", where the ordinary 4-dimensional space time manifold $M$ is replaced by a product $X=M \times F$, in which the space $F$ is not a manifold (unlike the case of the extra dimensions of string theory models) but a noncommutative space, which is metrically zero-dimensional but cohomologically (more precisely in terms of $K$-theoretic dimension) 6-dimensional. Thus, while spacetime itself does not acquire any amount of noncommutativity (unlike 
in the Moyal type compactifications of string theory), the presence of the "extra dimensions" given by the small noncommutative space $F$ determines the particle physics content of the model, in a way that is completely specified by the geometry of $F$. More precisely, the noncommutative space $X=M \times F$ is described by a spectral triple $(\mathcal{A}, \mathcal{H}, D)$, which is a product of the spectral triple describing the ordinary (commutative) Riemannian geometry of the spacetime manifold $M$ and of the spectral triple of the finite noncommutative space $F$ of the extra dimensions.

2.1. Spectral triples. A spectral triple is defined by data $(\mathcal{A}, \mathcal{H}, D)$ consisting of

- an involutive algebra $\mathcal{A}$,

- a representation $\pi: \mathcal{A} \rightarrow \mathcal{B}(\mathcal{H})$ as bounded operators on a separable Hilbert space,

- a self adjoint operator $D$ on $\mathcal{H}$, with the following properties:

- $D$ has compact resolvent, $\left(1+D^{2}\right)^{-1 / 2} \in \mathcal{K}(\mathcal{H})$,

- the commutators $[a, D]$ are bounded $\forall a \in \mathcal{A}$,

- the spectral triple is even if there is a $\mathbb{Z} / 2$-grading on $\mathcal{H}$ such that $[\gamma, a]=0$ and $D \gamma=-\gamma D$,

- the spectral triple has a real structure if there is an antilinear isometry $J: \mathcal{H} \rightarrow \mathcal{H}$ with $J^{2}=\varepsilon, J D=\varepsilon^{\prime} D J$, and $J \gamma=\varepsilon^{\prime \prime} \gamma J$, where $\varepsilon, \varepsilon^{\prime}, \varepsilon^{\prime \prime} \in\{ \pm 1\}$ are specified by the following table, which determines the KO-dimension of the spectral triple:

\begin{tabular}{|c|rrrrrrrr|}
\hline $\mathbf{n}$ & 0 & 1 & 2 & 3 & 4 & 5 & 6 & 7 \\
\hline \hline$\varepsilon$ & 1 & 1 & -1 & -1 & -1 & -1 & 1 & 1 \\
$\varepsilon^{\prime}$ & 1 & -1 & 1 & 1 & 1 & -1 & 1 & 1 \\
$\varepsilon^{\prime \prime}$ & 1 & & -1 & & 1 & & -1 & \\
\hline
\end{tabular}

- the Hilbert space $\mathcal{H}$ is a bimodule for $\mathcal{A}$, with the action of the form $b^{0}=J b^{*} J^{-1}$, for $b \in \mathcal{A}$, satisfying $\left[a, b^{0}\right]=0$ for all $a, b \in \mathcal{A}$,

- the order one condition for the operator $D$ holds: $\left[[D, a], b^{0}\right]=0$ for all $a, b \in \mathcal{A}$.

2.2. The spectral action functional. Spectral triples have an associated action functional, the spectral action introduced in [17]. This is of the form $\operatorname{Tr}\left(f\left(D_{A} / \Lambda\right)\right)$, where $D_{A}$ is the Dirac operator of the spectral triple, twisted with an inner fluctuation, while the function $f$ is a smooth approximation to a cutoff function. The energy scale $\Lambda$ is introduced for dimensional reasons. It is with respect to $\Lambda$ that one computes the asymptotic expansion of the spectral action.

One can then modify the action functional by adding a more conventional fermionic term, so as to obtain

$$
\operatorname{Tr}\left(f\left(D_{A} / \Lambda\right)\right)+\frac{1}{2}\left\langle J \tilde{\xi}, D_{A} \tilde{\xi}\right\rangle
$$

The form of the fermionic term varies in different NCG models, depending on the KO-dimension of the finite spectral triple. The form shown here above is the one that is relevant in the case considered in [21], where the finite noncommutative space $F$ has KO-dimension six. In this case, the fermion fields are regarded as Grassmann variables, and $\tilde{\xi}$ denotes the projection onto the +1 eigenspace $\mathcal{H}^{+}$ of the grading $\gamma$. The twisted Dirac operator is of the form $D_{A}=D+A+\varepsilon^{\prime} J A J^{-1}$, where the twisting gauge potentials are given by self-adjoint elements $A=A^{*}=\sum_{k} a_{k}\left[D, b_{k}\right]$, with $a_{k}, b_{k} \in \mathcal{A}$.

The spectral action functional has an asymptotic expansion for large energies $\Lambda$, as shown in [17],

$$
\operatorname{Tr}(f(D / \Lambda)) \sim \sum_{k \in \mathrm{DimSp}^{+}} f_{k} \Lambda^{k} f|D|^{-k}+f(0) \zeta_{D}(0)+o(1)
$$

with $f_{k}=\int_{0}^{\infty} f(v) v^{k-1} d v$ and the integration given by residues of zeta function $\zeta_{D}(s)=\operatorname{Tr}\left(|D|^{-s}\right)$ at the positive points of the dimension spectrum of the spectral triple, that is, the set of poles of the zeta functions. 
2.3. The finite geometry and the field content of the particle physics model. The main result of [21] shows that, if one chooses as an ansatz for the finite noncommutative space $F$ the algebra

$$
\mathcal{A}_{L R}=\mathbb{C} \oplus \mathbb{H}_{L} \oplus \mathbb{H}_{R} \oplus M_{3}(\mathbb{C}),
$$

then the asymptotic expansion of the spectral action recovers the full Lagrangian of an extension of the Minimal Standard Model of elementary particle physics, which includes Majorana mass terms for right handed neutrinos and neutrino mixing.

The main step of the construction of [21] go roughly as follows:

- The representation of the algebra $\mathcal{A}_{L R}$ is obtained by taking the sum $\mathcal{M}_{F}$ of all the inequivalent irreducible odd $\mathcal{A}_{L R}$-bimodules. One needs to fix as input the number $N$ of particle generations, which is done by choosing as Hilbert space of the finite geometry $\mathcal{H}_{F}=\oplus^{N} \mathcal{M}_{F}$. This provides the set of fermion fields of the particle physics model.

- The left-right chirality symmetry implicit in the choice of the algebra $\mathcal{A}_{L R}$ is broken when one imposes the order one condition for the Dirac operator. This selects a maximal subalgebra on which the condition holds, while still allowing the Dirac operator to mix the matter and antimatter sectors. The subalgebra is of the form $\mathcal{A}_{F}=\mathbb{C} \oplus \mathbb{H} \oplus M_{3}(\mathbb{C})$.

- The resulting noncommutative space $F$ is metrically zero dimensional but it has KO-dimension equal to 6.

- The real structure involution $J_{F}$ exchanges matter and antimatter, and the grading $\gamma_{F}$ distinguished the left and right chirality of particles.

- There is a complete classification of all the possible Dirac operators on this finite geometry, which is described in terms of geometric moduli spaces.

The last property listed here has a nice consequence: all the parameters of the particle physics model that correspond to Yukawa parameters of masses and mixing angles, as well as the additional Majorana mass terms for the right handed neutrinos, acquire a geometric meaning as coordinates on the moduli space of Dirac operators on the finite geometry $F$.

Let us first describe more closely the field content of the model following [21]. The fermions come from the basis of the Hilbert space $\mathcal{H}_{F}$,

$$
|\uparrow\rangle \otimes \mathbf{3}^{0}, \quad|\downarrow\rangle \otimes \mathbf{3}^{0}, \quad|\uparrow\rangle \otimes \mathbf{1}^{0}, \quad|\downarrow\rangle \otimes \mathbf{1}^{0} .
$$

These are matched with the particles (respectively, the up, charm and top quarks; the down, strange and bottom quarks; the $\nu_{e}, \nu_{\mu}$ and $\nu_{\tau}$ neutrinos; and the e $\mu$ and $\tau$ charged leptons) by computing the hypercharges, through the adjoint action of the $U(1)$ subgroup of the gauge group of the model, which is shown in [21] to be $U(1) \times S U(2) \times S U(3)$, up to finite abelian groups. The hypercharges are also computed in [21]. One obtains

$\begin{array}{ccccc} & \uparrow \otimes \mathbf{1}^{0} & \downarrow \otimes \mathbf{1}^{0} & \uparrow \otimes \mathbf{3}^{0} & \downarrow \otimes \mathbf{3}^{0} \\ \mathbf{2}_{L} & -1 & -1 & \frac{1}{3} & \frac{1}{3} \\ \mathbf{2}_{R} & 0 & -2 & \frac{4}{3} & -\frac{2}{3}\end{array}$

Since this gives the correct hypercharges, it justifies the identification of the chosen basis of $\mathcal{H}_{F}$ with the fermions. One then uses this basis in computing the explicit form of the Dirac operator.

2.4. Geometrization of Yukawa and Majorana parameters. It is then shown in [21] that the Dirac operator on the finite space $F$ is necessarily of the form

$$
D(Y)=\left(\begin{array}{cc}
S & T^{*} \\
T & \bar{S}
\end{array}\right), \quad S=S_{1} \oplus\left(S_{3} \otimes 1_{3}\right), \quad T=Y_{R}:\left|\nu_{R}\right\rangle \rightarrow J_{F}\left|\nu_{R}\right\rangle
$$




$$
\begin{aligned}
S_{1} & =\left(\begin{array}{cccc}
0 & 0 & Y_{\nu}^{*} & 0 \\
0 & 0 & 0 & Y_{\ell}^{*} \\
Y_{\nu} & 0 & 0 & 0 \\
0 & Y_{\ell} & 0 & 0
\end{array}\right) \\
S_{3} & =\left(\begin{array}{cccc}
0 & 0 & Y_{u}^{*} & 0 \\
0 & 0 & 0 & Y_{d}^{*} \\
Y_{u} & 0 & 0 & 0 \\
0 & Y_{d} & 0 & 0
\end{array}\right),
\end{aligned}
$$

where, for $N=3$ generations, the matrices in $\mathrm{GL}_{3}(\mathbb{C})$ that appear in this larger matrix are identified with Yukawa parameters as

- $Y_{\ell}$ : charged leptons, masses and mixing angles

- $Y_{\nu}$ : neutrinos, Dirac masses

- $Y_{d}$ : down/strange/bottom quark masses and mixing angles

- $Y_{u}$ up/charm/top quark masses

- $M=Y_{R}^{t}$ : symmetric matrix of Majorana mass terms for the right handed neutrinos

The moduli space of Dirac operators

$$
\mathcal{C}_{3} \times \mathcal{C}_{1}
$$

where $\mathcal{C}_{3}$ includes the parameters of the quark sector and $\mathcal{C}_{1}$ those of the lepton sector, is then described as a quotient of Lie groups

$$
\mathcal{C}_{3}=(K \times K) \backslash(G \times G) / K
$$

with $G=\mathrm{GL}_{3}(\mathbb{C})$ and $K=U(3)$. Here one identifies $\mathcal{C}_{3}$ with pairs $\left(Y_{d}, Y_{u}\right)$ modulo $W_{j}$ unitary matrices acting by

$$
Y_{d}^{\prime}=W_{1} Y_{d} W_{3}^{*}, Y_{u}^{\prime}=W_{2} Y_{u} W_{3}^{*}
$$

Thus, the number of real parameter accounted for by the quark sector of the model is $\operatorname{dim}_{\mathbb{R}} \mathcal{C}_{3}=10=$ $3+3+4$, accounting for mass eigenvalues, mixing angles, and CP-violating phase.

The case of the lepton sector is similar. One describes $\mathcal{C}_{1}$ in terms of triplets $\left(Y_{\ell}, Y_{\nu}, Y_{R}\right)$, with $Y_{R}$ a symmetric matrix, modulo the action of unitaries

$$
\begin{gathered}
Y_{\ell}^{\prime}=V_{1} Y_{\ell} V_{3}^{*}, Y_{\nu}^{\prime}=V_{2} Y_{\nu} V_{3}^{*}, \\
Y_{R}^{\prime}=V_{2} Y_{R} \bar{V}_{2}^{*}
\end{gathered}
$$

There is a projection $\pi: \mathcal{C}_{1} \rightarrow \mathcal{C}_{3}$ onto a space isomorphic to $\mathcal{C}_{3}$, which is obtained by forgetting $Y_{R}$. The fiber of the projection is the space of symmetric matrices modulo $Y_{R} \mapsto \lambda^{2} Y_{R}$. The dimension of the fiber is then $12-1=11$.

Thus, the total number of Yukawa parameters and Majorana mass terms in the model of [21] is $\operatorname{dim}_{\mathbb{R}}\left(\mathcal{C}_{3} \times \mathcal{C}_{1}\right)=31$.

A more general and detailed analysis of the moduli spaces of all the possible Dirac operators for finite geometries of the type considered in the construction of these particle physics models was given in [13].

2.5. Boson fields. The boson fields of the model come instead from the inner fluctuations of the Dirac operator. For self-adjoint gauge potentials of the form $A=\sum_{j} a_{j}\left[D, b_{j}\right]$ on the product geometry $M \times F$, one distinguishes fluctuations in the manifold direction $M$ and in the extra dimensions direction $F$. These give rise, respectively, to the gauge bosons and the Higgs field. Again, the identification with the $U(1), S U(2)$ and $S U(3)$ gauge bosons is confirmed by checking the quantum numbers. The Higgs sector predicted by this model consisting of a single Higgs doublet.

It is interesting to observe that, while the fermion content of the model is determined uniquely by the form of the finite geometry $F$, the boson content is specified only when the product geometry $M \times F$ is considered, since gauge bosons only arise as inner fluctuations in the $M$ directions.

A first estimate of the Higgs mass within this model was given in [21], using renormalization group analysis based on the RGE for the minimal Standard Model. It predited a heavy Higgs at around 170 
GeV. The recent results of [45] suggest that a broader range of Higgs estimates are possible within this model, when the RGEs for the minimal Standard Model are replaced by those for the extension with right handed neutrinos and Majorana mass terms, treated as in [1]. One can obtain in this way lighter Higgs estimates, below or around $158 \mathrm{GeV}$, and a broader spectrum of Higgs masses is likely available, due to the dependence on the initial conditions at unification of the RG flow.

The field content of the model, including the neutrino sector, agrees with the one predicted by the $\nu$ MSM model ( $c f .[58])$, where in addition to the parameter of the minimal SM there are additional real parameters that correspond to Majorana neutrino masses, and additional Yukawa coupling parameters for the lepton sector given by Dirac neutrino masses, mixing angles, and CP-violating phases.

A significant difference with respect to the $\nu \mathrm{MSM}$ model lies in the fact that the model of [21] has a preferred energy scale, which corresponds to unification of the coupling constants. One can see this by computing the asymptotic expansion (2.1) for large $\Lambda$ of the spectral action $\operatorname{Tr}\left(f\left(D_{A} / \Lambda\right)\right.$ ), as we discuss in $\S 2.7$.

2.6. The supersymmetric extension of the NCG standard model. Clearly, although the idea of using noncommutative geometry as a source of mathematical models for particle physics has been around since the mid 1990s, the potential model building capacities of this approach have not been fully developed yet. In view of the current high interest in model building in high energy physics in view of upcoming LHC data, as well as the expected new generation of cosmological data, it is the best possible time to attempt to extend the existing theoretical model.

The difficulty in doing so lies in the fact that, as we described above, the Lagrangian is computed from the geometry, so that it is very rigid and incorporating new fields can only be done through changes in the geometry, which are difficult to implement in just the right way.

However, a very important extension of the model of [21] was obtained recently in the paper by Thijs van den Broek and Walter van Suijlekom [9], which includes supersymmetric QCD.

The difficulty in extending the model to include the supersymmetric partners of standard model particles lies in the fact that one needs to enlarge the available inner fluctuations of the Dirac operator to incorporate the supersymmetric partners of the fermions, while at the same time not altering drastically the gauge symmetries of the model. This was achieved in [9] by changing the representation $\mathcal{H}_{F}$ in such a way as to obtain more inner fluctuations in the "vertical direction" $F$, which, in addition to the Higgs field, provide also the supersymmetric partners of the quarks, the squarks, again with the correct quantum numbers, and also allowing for the necessary extra fermions in the basis of $\mathcal{H}_{F}$, which give the superpartners of the gluons, the gluinos.

These new and very exciting results (it was previously erroneously believed that this type of particle physics models based on noncommutative geometry could not accommodate supersymmetry) still do not account for the full MSSM, the minimal supersymmetric standard model, because of the additional difficulties arising in the electroweak sector. However, the work of [9] has certainly identified the correct mechanism for implementing supersymmetry in the NCG models, and it becomes perfectly feasible to implement it across the full extent of the Standard Model.

2.7. Asymptotic expansion and unification energy. As we mentioned above, the full action functional considered in [21] includes the spectral action as well as an additional fermionic term,

$$
\operatorname{Tr}\left(f\left(D_{A} / \Lambda\right)\right)+\frac{1}{2}\left\langle J \tilde{\xi}, D_{A} \tilde{\xi}\right\rangle .
$$

The fermionic term is an antisymmetric bilinear form $\mathfrak{A}(\tilde{\xi})$ defined over $\mathcal{H}^{+}=\{\xi \in \mathcal{H} \mid \gamma \xi=\xi\}$, due to the sign involved in the commutation relation of $J$ and $D$ for KO-dimension six. It is nonvanishing on Grassmann variables and the corresponding Euclidean functional integral delivers a Pfaffian term

$$
P f(\mathfrak{A})=\int e^{-\frac{1}{2} \mathfrak{A}(\tilde{\xi})} D[\tilde{\xi}] .
$$

This reduces the counting of degrees of freedom with respect to the original model of [25], which was based on a finite noncommutative space of KO-dimension zero, hence it avoids a well known 
Fermion doubling problem of previous noncommutative geometry models of particle physics. The explicit calculation given in [21] of the fermionic term reproduces all the terms in the Standard Model Lagrangian (extended with right handed neutrinos with Majorana masses) that involve fermionic fields:

- the part $\mathcal{L}_{H f}$ of the Lagrangian involving the coupling of the Higgs to fermions,

- the part $\mathcal{L}_{g f}$ of the Lagrangian involving the coupling of gauge bosons to fermions,

- the part $\mathcal{L}_{f}$ of the Lagrangian involving only fermion fields.

In view of cosmological applications, however, the most interesting part of the action functional is the bosonic part given by $\operatorname{Tr}\left(f\left(D_{A} / \Lambda\right)\right)$, since that contains the gravitational information and the coupling of gravity to matter. Thus, we focus here mostly on this part and neglect the fermonic terms described above.

The asymptotic expansion (2.1) for large $\Lambda$ of the spectral action $\operatorname{Tr}\left(f\left(D_{A} / \Lambda\right)\right)$ was computed explicitly in [21] and it consists of the following terms:

$$
\begin{aligned}
\operatorname{Tr}\left(f\left(D_{A} / \Lambda\right)\right) \sim & \frac{1}{\pi^{2}}\left(48 f_{4} \Lambda^{4}-f_{2} \Lambda^{2} \mathfrak{c}+\frac{f_{0}}{4} \mathfrak{d}\right) \int \sqrt{g} d^{4} x \\
& +\frac{96 f_{2} \Lambda^{2}-f_{0} \mathfrak{c}}{24 \pi^{2}} \int R \sqrt{g} d^{4} x \\
& +\frac{f_{0}}{10 \pi^{2}} \int\left(\frac{11}{6} R^{*} R^{*}-3 C_{\mu \nu \rho \sigma} C^{\mu \nu \rho \sigma}\right) \sqrt{g} d^{4} x \\
& +\frac{\left(-2 \mathfrak{a} f_{2} \Lambda^{2}+\mathfrak{e} f_{0}\right)}{\pi^{2}} \int|\varphi|^{2} \sqrt{g} d^{4} x \\
& +\frac{f_{0} \mathfrak{a}}{2 \pi^{2}} \int\left|D_{\mu} \varphi\right|^{2} \sqrt{g} d^{4} x \\
& -\frac{f_{0} \mathfrak{a}}{12 \pi^{2}} \int R|\varphi|^{2} \sqrt{g} d^{4} x \\
& +\frac{f_{0} \mathfrak{b}}{2 \pi^{2}} \int|\varphi|^{4} \sqrt{g} d^{4} x \\
& +\frac{f_{0}}{2 \pi^{2}} \int\left(g_{3}^{2} G_{\mu \nu}^{i} G^{\mu \nu i}+g_{2}^{2} F_{\mu \nu}^{\alpha} F^{\mu \nu \alpha}+\frac{5}{3} g_{1}^{2} B_{\mu \nu} B^{\mu \nu}\right) \sqrt{g} d^{4} x
\end{aligned}
$$

where, in addition to the scalar curvature, one has other expressions of the curvature tensor, such as the Weyl curvature $C_{\mu \nu \rho \sigma}$, and the form that computes the Pontrjagin class,

$$
R^{*} R^{*}:=R_{\mu \nu \rho \sigma} R^{\mu \nu \rho \sigma}-4 R_{\mu \nu} R^{\mu \nu}+R^{2} .
$$

The expression above also contains several parameters, which play a very important role in our approach to cosmological models. In particular, there are two kinds of parameters involved:

- The constants $f_{0}, f_{2}, f_{4}$ are free parameters of the model, which arise in the calculation as momenta of the test function $f$ in the spectral action functional, $f_{0}=f(0)$ and, for $k>0$,

$$
f_{k}=\int_{0}^{\infty} f(v) v^{k-1} d v
$$

The value of the parameter $f_{0}$ will be related to the values of the coupling constants at unification, while the remaining two parameters $f_{2}$ and $f_{4}$ remain free parameters of the model. 
- The expressions $\mathfrak{a}, \mathfrak{b}, \mathfrak{c}, \mathfrak{d}, \mathfrak{e}$ are functions of the Yukawa parameters and Majorana mass terms of the model. They are explicitly given by the expressions

$$
\begin{aligned}
\mathfrak{a} & =\operatorname{Tr}\left(Y_{\nu}^{\dagger} Y_{\nu}+Y_{e}^{\dagger} Y_{e}+3\left(Y_{u}^{\dagger} Y_{u}+Y_{d}^{\dagger} Y_{d}\right)\right) \\
\mathfrak{b} & =\operatorname{Tr}\left(\left(Y_{\nu}^{\dagger} Y_{\nu}\right)^{2}+\left(Y_{e}^{\dagger} Y_{e}\right)^{2}+3\left(Y_{u}^{\dagger} Y_{u}\right)^{2}+3\left(Y_{d}^{\dagger} Y_{d}\right)^{2}\right) \\
\mathfrak{c} & =\operatorname{Tr}\left(M M^{\dagger}\right) \\
\mathfrak{d} & =\operatorname{Tr}\left(\left(M M^{\dagger}\right)^{2}\right) \\
\mathfrak{e} & =\operatorname{Tr}\left(M M^{\dagger} Y_{\nu}^{\dagger} Y_{\nu}\right) .
\end{aligned}
$$

- The coefficients of the terms in the asymptotic expansion (2.2) also depend explicitly on the energy scale $\Lambda$. A further dependence on the energy scale is hidden in the fact that the Yukawa parameters and Majorana masses, hence the functions (2.3) in turn run with the energy scale according to the renormalization group flow of the particle physics model, hence they in turn depend on $\Lambda$.

Let us digress momentarily to make an important remark on the last observation in the list of properties here above. It is customary, within the literature on noncommutative geometry models for particle physics, to assume that the relation expressed above, relating the coefficients of the asymptotic expansion of the spectral action to the Yukawa parameters of the particle physics content of the model, only holds at unification energy and does not transport in the same form to other energies, via the renormalization group. Thus, for instance, in [21] the running of the gravitational parameters of the model is analyzed assuming the relations above as boundary conditions at unification, and then the usual results, such as [31], that predict a very moderate or essential lack of running of the effective gravitational and cosmological constant. However, as noted in [45], one obtains a broader spectrum of possibilities within the model by considering also a scenario where the effective gravitational and cosmological constants of the model run, in the period of the very early universe that predates the electroweak epoch, according to the relation with the Yukawa parameters described above. We argue here below that the results obtained by considering this hypothesis are interesting enough to be worth pursuing this line of investigation. In particular, we argue that this latter property, the running of the coefficients (2.3) with the renormalization group flow, is particular to only this type of particle physics model, and it has a series of direct and interesting consequences on related cosmological models of the primordial universe, as developed in [45]. We return to discuss this in detail in $\S 3$ below.

The standard normalization of the Yang-Mills terms and the Higgs kinetic term in (2.1) leads to an effective action of form

$$
\begin{aligned}
S & =\frac{1}{2 \kappa_{0}^{2}} \int R \sqrt{g} d^{4} x+\gamma_{0} \int \sqrt{g} d^{4} x \\
& +\alpha_{0} \int C_{\mu \nu \rho \sigma} C^{\mu \nu \rho \sigma} \sqrt{g} d^{4} x+\tau_{0} \int R^{*} R^{*} \sqrt{g} d^{4} x \\
& +\frac{1}{2} \int|D H|^{2} \sqrt{g} d^{4} x-\mu_{0}^{2} \int|H|^{2} \sqrt{g} d^{4} x \\
& -\xi_{0} \int R|H|^{2} \sqrt{g} d^{4} x+\lambda_{0} \int|H|^{4} \sqrt{g} d^{4} x \\
& +\frac{1}{4} \int\left(G_{\mu \nu}^{i} G^{\mu \nu i}+F_{\mu \nu}^{\alpha} F^{\mu \nu \alpha}+B_{\mu \nu} B^{\mu \nu}\right) \sqrt{g} d^{4} x .
\end{aligned}
$$


The coefficients of this normalized form of the bosonic Lagrangian obtained from the asymptotic expansion of the spectral action are then given by

$$
\begin{aligned}
\frac{1}{2 \kappa_{0}^{2}} & =\frac{96 f_{2} \Lambda^{2}-f_{0} \mathfrak{c}}{24 \pi^{2}} & \gamma_{0} & =\frac{1}{\pi^{2}}\left(48 f_{4} \Lambda^{4}-f_{2} \Lambda^{2} \mathfrak{c}+\frac{f_{0}}{4} \mathfrak{d}\right) \\
\alpha_{0} & =-\frac{3 f_{0}}{10 \pi^{2}} & \tau_{0} & =\frac{11 f_{0}}{60 \pi^{2}} \\
\mu_{0}^{2} & =2 \frac{f_{2} \Lambda^{2}}{f_{0}}-\frac{\mathfrak{e}}{\mathfrak{a}} & \xi_{0} & =\frac{1}{12} \\
\lambda_{0} & =\frac{\pi^{2} \mathfrak{b}}{2 f_{0} \mathfrak{a}^{2}} . & &
\end{aligned}
$$

One sees, in this form, that the spectral action is considered at a preferred energy scale where

$$
\frac{g^{2} f_{0}}{2 \pi^{2}}=\frac{1}{4}
$$

gives the value $g$ at unification of the coupling constants for the gauge fields (i.e. all the coupling constants are taken to be equal at this scale). In particle physics models one assumes the unification energy to be located somewhere in the range of $10^{15}-10^{17} \mathrm{GeV}$.

Thus, when one discusses renormalization group analysis of the asymptotic form of the spectral action, which is the technique by which one obtains within this type of model lower energy predictions such as the Higgs mass, one assumes that initial conditions for the RGE flow are assigned at unification energy, based on constraints imposed by the geometry of the model, and then the RGE flow is computed downward towards lower energies and the electroweak scale.

This type of renormalization group analysis is the basis for the early universe models developed in [45], which we describe in the next section.

\section{EARly UNIVERSE MODELS AND RGE ANALYSIS}

In this section I will review the results of the joint work with Elena Pierpaoli [45] and the analysis of the boundary conditions of the model at unification energy performed in joint work with Daniel Kolodrubetz in [38].

3.1. Gravitational terms and cosmology. The asymptotic expansion of the spectral action contains the usual gravitational and cosmological terms of general relativity, namely the Einstein-Hilbert action

and the cosmological term

$$
\frac{1}{2 \kappa_{0}^{2}} \int R \sqrt{g} d^{4} x
$$

$$
\gamma_{0} \int \sqrt{g} d^{4} x
$$

although with an unusual form of the coefficients, about which we are going to say more below. It also contains some additional terms, like the non-dynamical topological term

$$
\tau_{0} \int R^{*} R^{*} \sqrt{g} d^{4} x
$$

which integrates to a multiple of the Euler characteristic of the spacetime manifold, and a conformal gravity term

$$
\alpha_{0} \int C_{\mu \nu \rho \sigma} C^{\mu \nu \rho \sigma} \sqrt{g} d^{4} x,
$$

which is given in terms of the Weyl curvature tensor and a conformal coupling of the Higgs field to gravity

$$
\xi_{0} \int R|H|^{2} \sqrt{g} d^{4} x .
$$


The remaining terms are the usual kinetic, mass, and quartic self-interaction of the Higgs field, and Yang-Mills terms for the gauge bosons.

In terms of the cosmological timeline, one has the following correspondence between cosmological eras and energy scales [36]:

- Planck epoch: $t \leq 10^{-43} s$ after the Big Bang (unification of forces with gravity, the realm of quantum gravity)

- Grand Unification epoch: $10^{-43} s \leq t \leq 10^{-36} s$ (electroweak and strong forces unified; Higgs)

- Electroweak epoch: $10^{-36} s \leq t \leq 10^{-12} s$ (strong and electroweak forces separated)

- Inflationary epoch: possibly $10^{-36} s \leq t \leq 10^{-32} s$

One sees from this timeline chart that the unification energy corresponds to a time in the very early universe, around $10^{-36} \mathrm{~s}$ after the Big Bang. Thus, whatever cosmological observations one derives directly from the asymptotic expansion of the spectral action refer to a time that far predates anything that is accessible by direct observation. In fact, even when one uses renormalization group techniques to run down the energy scale from specified initial condition at unification to the electroweak scale, where the next phase transition happens, one still remains within the realm of the primordial universe. In fact, the electroweak transition is still taking place at a time of around $10^{-12} \mathrm{~s}$. However, this range of energies, or correspondingly this range of times, is especially interesting in theoretical cosmology, due to the fact that the inflationary epoch is supposed to have taken place somewhere in between the unification and the electroweak scales. This suggests that the asymptotic expansion of the spectral action can be studied as a possible source of inflation scenarios.

The main features of the asymptotic expansion of the spectral action (2.1) that will play a role in the cosmological models of the very early universe and the inflationary epoch are the fact that the model contains an effective gravitational constant given by

$$
G_{\text {eff }}=\frac{\kappa_{0}^{2}}{8 \pi}=\frac{3 \pi}{192 f_{2} \Lambda^{2}-2 f_{0} \mathfrak{c}(\Lambda)}
$$

and an effective cosmological constant given by

$$
\gamma_{0}=\frac{1}{4 \pi^{2}}\left(192 f_{4} \Lambda^{4}-4 f_{2} \Lambda^{2} \mathfrak{c}(\Lambda)+f_{0} \mathfrak{d}(\Lambda)\right),
$$

both of which can be considered as functions of the energy scale, through the explicit dependence on $\Lambda$, as well as through the implicit $\Lambda$-dependence of the functions $\mathfrak{c}$ and $\mathfrak{d}$ of (2.3), via their running with the renormalization group flow determined by the particle physics content of the model of [21].

Some of the main cosmological implications of the model described in [45] are:

- Linde's hypothesis (antigravity in the early universe)

- Primordial black holes and gravitational memory

- Gravitational waves in modified gravity

- Gravity balls

- Varying effective cosmological constant

- Higgs based slow-roll inflation

- Spontaneously arising Hoyle-Narlikar in EH backgrounds

We will focus here on those aspects that look more promising in terms of possible predictions that can be testable against observable cosmological data.

3.2. Renormalization group equations. For the particle physics model obtained from the asymptotic expansion (2.1) of the spectral action, whose field content is given by an extension of the standard model with right handed neutrinos with Majorana mass terms, one can compute explicitly the renormalization group equations at 1-loop. This was done in [1], for this type of extension of the Standard Model as well as for other extensions that also involve supersymmetry. In the case which is directly of interest for the particle physics model derived in [21] the renormalization group equations of [1] at 
1-loop order are given by the $\beta$ functions

$$
\begin{gathered}
16 \pi^{2} \beta_{g_{i}}=b_{i} g_{i}^{3} \quad \text { with }\left(b_{S U(3)}, b_{S U(2)}, b_{U(1)}\right)=\left(-7,-\frac{19}{6}, \frac{41}{10}\right) \\
16 \pi^{2} \beta_{Y_{u}}=Y_{u}\left(\frac{3}{2} Y_{u}^{\dagger} Y_{u}-\frac{3}{2} Y_{d}^{\dagger} Y_{d}+\mathfrak{a}-\frac{17}{20} g_{1}^{2}-\frac{9}{4} g_{2}^{2}-8 g_{3}^{2}\right) \\
16 \pi^{2} \beta_{Y_{d}}=Y_{d}\left(\frac{3}{2} Y_{d}^{\dagger} Y_{d}-\frac{3}{2} Y_{u}^{\dagger} Y_{u}+\mathfrak{a}-\frac{1}{4} g_{1}^{2}-\frac{9}{4} g_{2}^{2}-8 g_{3}^{2}\right) \\
16 \pi^{2} \beta_{Y_{\nu}}=Y_{\nu}\left(\frac{3}{2} Y_{\nu}^{\dagger} Y_{\nu}-\frac{3}{2} Y_{e}^{\dagger} Y_{e}+\mathfrak{a}-\frac{9}{20} g_{1}^{2}-\frac{9}{4} g_{2}^{2}\right) \\
16 \pi^{2} \beta_{Y_{e}}=Y_{e}\left(\frac{3}{2} Y_{e}^{\dagger} Y_{e}-\frac{3}{2} Y_{\nu}^{\dagger} Y_{\nu}+\mathfrak{a}-\frac{9}{4} g_{1}^{2}-\frac{9}{4} g_{2}^{2}\right) \\
16 \pi^{2} \beta_{M}=Y_{\nu} Y_{\nu}^{\dagger} M+M\left(Y_{\nu} Y_{\nu}^{\dagger}\right)^{T} \\
16 \pi^{2} \beta_{\lambda}=6 \lambda^{2}-3 \lambda\left(3 g_{2}^{2}+\frac{3}{5} g_{1}^{2}\right)+3 g_{2}^{4}+\frac{3}{2}\left(\frac{3}{5} g_{1}^{2}+g_{2}^{2}\right)^{2}+4 \lambda \mathfrak{a}-8 \mathfrak{b} .
\end{gathered}
$$

Because the additional Majorana mass terms for right handed neutrinos introduce non-renormalizable interactions, the way to deal with this renormalization group equations, as explained in [1] is to apply them to different effective field theories in between the different see-saw scales of the model.

One works under the assumption of having a non-degenerate spectrum of Majorana masses, which determine three see-saw scales in between the unification and the electroweak scale, which correspond to the three Majorana masses. Typically, one assumes that the top Majorana mass is close to the unification scale, which suffices to have the see-saw mechanism in the model which ensures the necessary smallness of the light neutrino masses. In between the different see-saw scales one consider different effective field theories where the highest modes are progressively integrated out as one moves down to lower energies and passes through one of the see-saw scales scales. Thus, one proceeds as follows:

- identify suitable initial conditions for the RGE flow at unification energy $\Lambda_{\text {unif }}$;

- run the RGE flow down from unification $\Lambda_{\text {unif }}$ to first see-saw scale, which is the largest eigenvalue of $M$;

- introduce the next effective field theory, with the matrix $Y_{\nu}^{(3)}$ obtained by removing the last row of $Y_{\nu}$ in the basis in which $M$ diagonal, and the matrix $M^{(3)}$ obtained by removing the last row and column of $M$;

- run the induced RGE flow with matching boundary conditions at the top see-saw scale down to second see-saw scale;

- introduce the next effective field theory, with $Y_{\nu}^{(2)}$ and $M^{(2)}$, with matching boundary conditions at the second see-saw scale;

- run the induced RGE flow down to the smallest see-saw scale;

- introduce the next effective field theory with $Y_{\nu}^{(1)}$ and $M^{(1)}$ and matching boundary conditions at the lowest see-saw scale;

- run the induced RGE flow down to the electoweak energy $\Lambda_{e w}$.

3.3. Conformal coupling of Higgs and gravity and slow-roll inflation. One seeks, within our model, predictions that can be tested directly against observational cosmological data. This is made especially complicated by the fact that the asymptotic expansion of the spectral action lives at energies close to unification, while any accessible cosmological data comes from much more recent eras of the universe (even when talking about data extracted from the cosmic microwave background radiation, which is the farthest observationally accessible surface in the universe). Thus, one needs to identify properties of the very early universe that can leave a detectable signature in the CMB or in the more modern universe.

A good part of the recent spectacular results in theoretical and observational cosmology focused on the fact that certain signatures of possible inflation scenarios can in fact be detected in the CMB. One such example is the fact that one can obtain very strong constraints from observational data 
about the CMB on certain quantities, called spectral index and tensor-to-scalar ratio, which in turn, in slow-roll inflation models, are determined by the shape of the potential of the field that runs the inflation. In view of this type of signatures of early universe models, we sought to understand what predictions one can derive within our model about these parameters.

The presence in the asymptotic expansion of the spectral action of a non-minimal conformal coupling of the Higgs field to gravity

$$
\frac{1}{16 \pi G_{\mathrm{eff}}} \int R \sqrt{g} d^{4} x-\frac{1}{12} \int R|H|^{2} \sqrt{g} d^{4} x
$$

allows for the development, within the model, of slow-roll inflation scenarios based on the Higgs field.

Slow-roll potentials are a very well known mechanism to produce inflation scenarios in theoretical cosmology. The specific form of the potential depends on the specific particle physics content of the model and on the resulting field $\phi$ that is responsible for the inflation mechanism. The general setting is the following. Consider a 4-dimensional Minkowskian spacetime which is a cylinder over a 3-dimensional Riemannian manifold $X$, with the Friedmann metric

$$
d s^{2}=a(t)^{2} d s_{X}^{2}-d t^{2}
$$

The factor $a(t)$ drives the expansion. In the case of a slow-roll potential $V(\phi)$ for a scalar field $\phi$, the accelerated expansion $\frac{\ddot{a}}{a}=H^{2}(1-\epsilon)$ depends on the Hubble parameter, which in turn is given by

$$
H^{2}(\phi)\left(1-\frac{1}{3} \epsilon(\phi)\right)=\frac{8 \pi}{3 m_{P l}^{2}} V(\phi),
$$

with $m_{P l}$ the Planck mass and the first slow-roll parameter

$$
\epsilon(\phi)=\frac{m_{P l}^{2}}{16 \pi}\left(\frac{V^{\prime}(\phi)}{V(\phi)}\right)^{2}
$$

The inflationary phase happens when $\epsilon(\phi)<1$. One also has a second slow-roll parameter

$$
\eta(\phi)=\frac{m_{P l}^{2}}{8 \pi}\left(\frac{V^{\prime \prime}(\phi)}{V(\phi)}\right)-\frac{m_{P l}^{2}}{16 \pi}\left(\frac{V^{\prime}(\phi)}{V(\phi)}\right)^{2} .
$$

These two parameters determine measurable quantities, which can be tested in observational data on the CMB, namely the spectral index and tensor-to-scalar ratio, respectively given by the expressions

$$
n_{s}=1-6 \epsilon+2 \eta \quad \text { and } \quad r=16 \epsilon .
$$

While the mechanism recalled here, relating the potential $V(\phi)$ to the accelerated expansion of the scale factor $a(t)$, refers to a Minkowskian spacetime with a Friedmann metric, the potential itself continues to make sense in a Wick rotates and compactified Euclidean metric, for which it is suitable to use spectral action techniques. Thus, our strategy generally consist of arguing with the spectral action applied to a Riemannian metric and, once the correct inflation potential has been identified in this setting, to look at its cosmological consequences back in the Minkowskian case. This often requires making sure that the resulting calculation of the observable quantities coming from the slowroll potential is in fact independent of the choices made in the Euclidean compactification. We discuss this in more detail in $\S 4$ below.

Slow-roll inflation scenarios based on a coupling of Higgs to gravity of the form (3.3), with $G_{\text {eff }}=G$ the usual gravitational constant, were recently developed in [30]. The scenario one obtains in our model is essentially analogous to the one developed in [30], and this was observed also in the recent work [52] and [11]. However, while in [30] one considers the case of a non-conformal coupling, with $\xi_{0} \neq 1 / 12$ away from the conformal fixed point and running with the RG flow, but with fixed $G_{\text {eff }}=G$, in our model we find that $\xi_{0}=1 / 12$ stays fixed at the conformal fixed point, while the running of $G_{\text {eff }}$, given 
by (3.1), with the energy scale $\Lambda$ through the RGE flow of [1] reproduces the same shape of slow-roll potential as that found in [30]. One has, in our case

$$
V_{E}(x)=\frac{\lambda_{0} x^{4}}{\left(1+\xi_{0} \kappa_{0}^{2} x^{2}\right)^{2}}
$$

for which the spectral index and tensor to scalar ratio take the form

$$
\begin{gathered}
n_{s}=1+\frac{32\left(216+\kappa_{0}^{2}\left(6 x^{2}-\kappa_{0}^{2}\left(432+12 \kappa_{0}^{2}\left(2+3\left(\kappa_{0}^{2}\right)^{2}\right) x^{2}+\left(1+\left(\kappa_{0}^{2}\right)^{2}\right) x^{4}\right)\right)\right)}{\kappa_{0}^{2}\left(12 x+\kappa_{0}^{2}\left(1+\left(\kappa_{0}^{2}\right)^{2}\right) x^{3}\right)^{2}} \\
r=\frac{256 \kappa_{0}^{2}}{x^{2}+\frac{\kappa_{0}^{2}}{12}\left(1+\left(\kappa_{0}^{2}\right)^{2}\right) x^{4}}
\end{gathered}
$$

in terms of the parameter $\kappa_{0}^{2}$ of $(3.1)$.

In fact, as we showed in [46] (see $\S 4$ below), nonperturbative calculations of the spectral action, at least for some especially symmetric geometries, give a more refined estimate for the slow-roll parameters and the spectral index and tensor to scalar ratio.

3.4. Primordial black holes. There is another type of phenomenon that takes place in the very early universe and which may leave an observationally detectable signature in the modern universe, namely primordial black holes. These are not stellar black holes, since they formed in an epoch that far predates structure formation, but rather they are created by the collapse of overdense regions of space in the primordial universe. They are related to various important cosmological phenomena, such as phase transitions in the early universe, cosmic loops and strings, inflationary reheating, and they were introduced as a theoretical hypothesis in [56]. Although there is no conclusive evidence on the existence of primordial black holes, it is known that they would follow the same law of evaporation by Hawking radiation as their stellar counterparts. The evaporation law is given by

$$
\frac{d \mathcal{M}(t)}{d t} \sim-\left(G_{\text {eff }}(t) \mathcal{M}(t)\right)^{-2}
$$

and the Hawking temperature is $T=\left(8 \pi G_{\text {eff }}(t) \mathcal{M}(t)\right)^{-1}$.

The reason why primordial black holes may be especially relevant to our investigation lies in the fact that some primordial black holes may evaporate sufficiently slowly that they would persist until the more modern universe, hence providing a window to probe the more distant universe, well beyond the last scattering surface of the CMB.

In particular, as observed in [6], if one is looking at cosmological models where in the very early universe one has an effective gravitational constant which is variable and different from the value it has in the more modern universe, then the intriguing possibility arises of primordial black hole whose evaporation law either changes with the changing value of the effective gravitational constant or else whose evaporation law is dictated by the value of the effective gravitational constant at the time of their formation. This is called the phenomenon of gravitational memory. Either way, an altered evaporation law, with respect to the one that corresponds to the fixed gravitational constant of the modern universe, could leave detectable traces of phenomena arising in the primordial universe. In fact, the evaporation of primordial black holes is regarded as a possible model for gamma ray bursts, and there are experimental searches (see [12]) that can test different primordial black holes evaporation models.

In terms of the energy variable one can write the evaporation law as

$$
\mathcal{M}^{2} d \mathcal{M}=\frac{1}{\Lambda^{2} G_{\text {eff }}^{2}\left(\Lambda, f_{2}\right)} d \Lambda \text {. }
$$

Given the form of the effective gravitational constant (3.1) and its running with $\Lambda$ through the renormalization group equations of [1], we find that in our model one has a modified evaporation law for 
primordial black holes of the form

$$
\mathcal{M}\left(\Lambda, f_{2}\right)=\sqrt[3]{\mathcal{M}^{3}\left(\Lambda_{i n}\right)-\frac{2}{3 \pi^{2}} \int_{\Lambda}^{\Lambda_{i n}} \frac{\left(1-\frac{4 \pi}{3} G_{\text {eff }}(x)|H|^{2}\right)^{2}}{x^{3} G_{\text {eff }}(x)^{2}} d x}
$$

In order to employ in our NCG setting the methods of [12] to link this modified evaporation law of primordial black holes to models of gamma ray bursts, one needs to control the effect of correction terms from the low energy nonperturbative effects of the spectral action (see $\S 4$ below) and check how these affect the evaporation law obtained at high energies in [45], so that one can treat the case of those primordial black holes that did not completely evaporate until sufficently recent times in the universe to become significant as possible models of gamma ray bursts and therefore accessible through the analysis of [12].

3.5. Other effects: Gravity balls, gravitational waves, variable cosmological constant, Hoyle-Narlikar cosmologies. It was observed by Linde in [41] that, in the presence of a conformal coupling to gravity of the form

$$
\frac{1}{16 \pi G} \int R \sqrt{g} d^{4} x-\frac{1}{12} \int R \phi^{2} \sqrt{g} d^{4} x,
$$

one can obtain an effective gravitational constant of the form

$$
G_{\text {eff }}^{-1}=G^{-1}-\frac{4}{3} \pi \phi^{2}
$$

which may give rise to phenomena of "negative gravity" in the early universe, which in turn can also provide possible inflation mechanisms.

3.5.1. Gravity balls. In our model of the very early universe based on the asymptotic expansion of the spectral action, when one replaces (3.7) with (3.3), one obtains a refined version of the Linde mechanism, by which not only one can achieve regions of negative gravity through the same mechanism based on the coupling of gravity with the Higgs field, but one also has a variable effective gravitational constant to begin with in (3.3), which in turn contributes to achieving different possible shapes of regions and epochs of negative gravity, or "gravity balls". One finds that $G_{\text {eff }}(\Lambda)>0$ when

$$
\begin{cases}G_{\mathrm{eff}, H}<0 & \text { for }|H|^{2}>\frac{3}{4 \pi G_{\mathrm{eff}}(\Lambda)}, \\ G_{\mathrm{eff}, H}>0 & \text { for }|H|^{2}<\frac{3}{4 \pi G_{\mathrm{eff}}(\Lambda)},\end{cases}
$$

where $G_{\mathrm{eff}, H}$ is given by

$$
G_{\text {eff }, H}=G_{\text {eff }}\left(1-\frac{4 \pi}{3} G_{\text {eff }}|H|^{2}\right)^{-1} .
$$

The behavior of $G_{\text {eff }}$ and $G_{\text {eff }, H}$ with $\Lambda$ depend significantly on the choice of the free parameter $f_{2}$ of the model.

3.5.2. Gravitational waves. The variable effective gravitational constant in the early universe also affects the propagation of the gravitational waves. In fact, the Einstein equations $R^{\mu \nu}-\frac{1}{2} g^{\mu \nu} R=$ $\kappa_{0}^{2} T^{\mu \nu}$ for

$$
g_{\mu \nu}=a(t)^{2}\left(\begin{array}{cc}
-1 & 0 \\
0 & \delta_{i j}+h_{i j}(x)
\end{array}\right),
$$

when separating out the trace and traceless contributions give the Friedmann equation for the trace part

$$
-3\left(\frac{\dot{a}}{a}\right)^{2}+\frac{1}{2}\left(4\left(\frac{\dot{a}}{a}\right) \dot{h}+2 \ddot{h}\right)=\frac{\tilde{\kappa}_{0}^{2}}{\Lambda^{2}} T_{00}
$$

During the inflationary epoch the ansatz for the behavior of the expansion is given by assuming $a(t) \sim e^{\alpha t}$. With the usual relation between the energy scale and the expansion factor given by 
$\Lambda(t)=1 / a(t)$, one finds that in our noncommutative geometry model, for a choice of $f_{2}$ that recovers the usual Newton constant $G$ at low energies, one has solutions behaving like

$$
h(t)=\frac{3 \pi^{2} T_{00}}{192 f_{2} \alpha^{2}} e^{2 \alpha t}+\frac{3 \alpha}{2} t+\frac{A}{2 \alpha} e^{-2 \alpha t}+B,
$$

in contrast with the usual Einstein-Hilbert cosmology with fixed gravitational constant where one has

$$
h(t)=\left(\frac{4 \pi G T_{00}}{\alpha}+\frac{3 \alpha}{2}\right) t+\frac{A}{2 \alpha} e^{-2 \alpha t}+B .
$$

Thus, we see a phenomenon of amplification of the gravitational waves. This persists into the radiation dominated epoch, where $a(t) \sim t^{1 / 2}$, and one finds solutions of the form

$$
h(t)=\frac{4 \pi^{2} T_{00}}{288 f_{2}} t^{3}+B+A \log (t)+\frac{3}{8} \log (t)^{2}
$$

in our model in contrast to solutions of the form

$$
h(t)=2 \pi G T_{00} t^{2}+B+A \log (t)+\frac{3}{8} \log (t)^{2}
$$

in the ordinary case.

The problem with this effect on the gravitational waves is that it happens in the very early universe and one cannot expect that it will still be detectable in the modern universe. However, since there is a coupling of the gravitational waves and the CMB, which should be detectable in the polarization spectrum that the new Planck data are recording, it is worth investigating whether there is a way that predictions made in this model about gravitational waves that differ from the ordinary cosmology may have a detectable signature on such cosmological data. A more recent analysis of effects of the NCG model on the gravitational waves was carried out in [53].

3.5.3. Running effective cosmological constant. The running effective cosmological constant (3.2) of the model already by itself provides a possible inflation scenario, since one can chose initial conditions at unification for the RGE flow and values of the free parameters $f_{2}$ and $f_{4}$ of the model so that the effective cosmological constant becomes very small when one comes towards the modern universe, while being very large near the unification epoch.

3.5.4. Emergent Hoyle-Narlikar cosmologies. Another aspect of this model that depends on the running of the effective gravitational and cosmological constants is the presence of phase transitions in the early universe associated to the see-saw scales defined by the eigenvalues of the matrix of the Majorana mass terms. It is shown in [45] that the dominant term in the asymptotic expansion of the spectral action (2.1) is usually the ordinary Einstein-Hilbert term. However, one can see explicit examples, for suitable choices of the boundary conditions of the RGE flow at unification and of the free parameters $f_{2}$ and $f_{4}$ in the model, where near the phase transitions of the see-saw scales the Einstein-Hilbert term becomes subdominant and the dominant term that emerges near the phase transition is a Hoyle-Narlikar cosmology of the form

$$
\begin{aligned}
S_{c}= & \alpha_{0} \int C_{\mu \nu \rho \sigma} C^{\mu \nu \rho \sigma} \sqrt{g} d^{4} x+\frac{1}{2} \int|D H|^{2} \sqrt{g} d^{4} x \\
& -\xi_{0} \int R|H|^{2} \sqrt{g} d^{4} x+\lambda_{0} \int|H|^{4} \sqrt{g} d^{4} x \\
& +\frac{1}{4} \int\left(G_{\mu \nu}^{i} G^{\mu \nu i}+F_{\mu \nu}^{\alpha} F^{\mu \nu \alpha}+B_{\mu \nu} B^{\mu \nu}\right) \sqrt{g} d^{4} x .
\end{aligned}
$$


3.6. Boundary conditions at unification for the RG flow. I present here briefly the results of the recent joint paper with Daniel Kolodrubetz, [38].

A very important aspect of the results of [45] discussed above is the fact that there is a choice of boundary conditions at unification energy for the RGE flow, which in turns determines the behavior of the coefficients $\mathfrak{a}, \mathfrak{b}, \mathfrak{c}, \mathfrak{d}, \mathfrak{e}$ of $(2.3)$ and therefore of the effective gravitational and effective cosmological constants of (3.1) and (3.2).

Most of the analysis of [45] was carried out using the default boundary conditions for the RGE flow of [1]. These are compatible with experimental results at the low energy limit, but they are not fully compatible with all the constraints on the values at unification imposed by the geometry of the model, as described in [21].

In [38] a broader and more systematic analysis of the boundary conditions at unification was carried out. This revealed a strong dependence on the choice of the boundary conditions of the behavior of the coefficients (2.3), which makes the fine tuning problem for the values of the parameters at unification very delicate. For example, the behavior of the coefficients (2.3) is computed numerically for different choices of boundary conditions such as the "maximal mixing" case and compared to the default boundary conditions of [1] used in [45].

More importantly, a specific set of boundary conditions is identified in [38], which differ from the default choice of [1] and is obtained by combining the maximal mixing conditions with a suitably modified Majorana mass matrix and Higgs parameters at unification. This set of boundary conditions, which we refer to as modified maximal mixing conditions, is still compatible, within the same order of magnitude, with the expected experimental values at low energy, but which also satisfies all the geometric constraints of [21] at unification. The qualitative behavior of all the phenomena described in the previous section, as obtained in [45], is not significantly altered by using these new boundary conditions.

3.7. Supersymmetric versions. In terms of cosmological implications, the same approach followed in [45] to generate early universe models can be applied to any supersymmetric extensions of the current NCG model, such as those of [9] described in $\S 2.6$ above.

The entire RGE analysis will behave very differently in the presence of supersymmetric extensions, as the changes in the particle physics content of the model will reflect both on a different explicit form of the dependence on Yukawa parameters of the coefficients of the spectral action and on the different form of the 1-loop beta function and the renormalization group equations.

Moreover, the concrete possibility of obtaining models with supersymmetry opens up a wider range of possible dark matter models that can be implemented within this NCG framework, see $\S 5.1$ below.

Finally, the possibility of extending the NCG model to supersymmetric version makes more concrete and plausible the existing conjecture that the K-theoretically 6 -dimensional space $F$ may be a noncommutative low energy limit of a Calabi-Yau geometry of some string theory vacuum, see $\S 5.4$ below.

\section{NonPerturbative SPECTRAL ACTION AND COSMIC TOPOLOGY}

In this section I describe recently obtained results contained in the joint work with Elena Pierpaoli and Kevin Teh [46].

4.1. Moving towards lower energies, nonperturbative effects. One of the most important steps in extending cosmological models based on the NCG approach of [21] and possible generalizations from the very early universe to the more recent universe where one has a larger range of cosmological data available to test the model, is to understand and estimate the correction terms that enter from nonperturbative effects in the spectral action functional when its high-energy asymptotic expansion, which is used to produce the Lagrangian of the model, is compared to the spectral action itself. If one has a good control on what correction terms arise, and an estimate of their size and effect, one can 
extend the analysis of [45] to the more recent cosmological epochs of nucleosynthesis and structure formation and derive a much broader range of cosmological consequences of the model.

One does not expect that nonperturbative corrections will affect the matter content of the model, as already the high energy expansion contains the particle physics content of the Standard Model, but it may affect the behavior of the gravitational terms and the dependence of their coefficients upon the Yukawa parameters, which are in fact the most important features from the cosmological viewpoint.

The main difficulty in dealing with the spectral action itself, rather than its asymptotic expansion, is the fact that it is given by a global expression $\operatorname{Tr}\left(f\left(D_{A} / \Lambda\right)\right)$ involving the spectrum of the Dirac operator, which is usually not known exactly for arbitrary geometries. However, by restricting the model to only the gravitational sector (that is, considering only the ordinary manifold $M$ without the fiber noncommutative space $F$, and the ordinary Dirac operator on $M$ ) and by restricting attention to only some especially symmetric geometries, in a way similar to what was recently done in [20] in the sphere case, we were able in [46] to explicitly compute the spectral action non-perturbatively and identify specific nonperturbative effects that have direct consequences on the possible inflation scenarios allowed by the model. In particular, the results of [46] reveal the fact that this type of model exhibits a coupling of geometry and inflation, by which the type of geometry (spherical or flat) of the spatial hypersurfaces determines different possible forms of the slow-roll inflation potentials allowed in the model.

4.2. The problem of cosmic topology. The issue of a possibly nontrivial topological shape of the universe is a long standing problem. It cannot be answered by the Einstein equations, which would not distinguish between space hypersurfaces that are locally isometric manifolds with distinct topology, like a 3-torus instead of flat Euclidean 3-space, or the Poincaré homology sphere instead of the ordinary 3-sphere. It has become an area of active study, however, to investigate whether the presence of nontrivial topology can leave a detectable signature in the cosmological observations, most notably in the temperature and polarization spectra of the cosmic microwave radiation [57]. A method that seemed promising was to search statistically for "matching circles in the sky", i.e. for regions in the WMAP that would correspond to matching faces of a fundamental domain of a nontrivial 3-manifold. This type of search has been carried out for the Poincaré homology sphere and for the case of 3-tori, but despite some indication of possible matches the evidence is at present still inconclusive. Among the available cosmological data, what could be a possible indication of the presence of non-trivial topology, though not of what kind of topology, is the unexpected alignment of the quadrupole-octupole momenta, which is not explained in the simply connected case. Recent results show that the most carefully studied candidate of a possible non-trivial topology, the Poincaré homology sphere, also fails to account for this alignment [62]. Other possible candidate 3-dimensional manifolds have been analyzed, especially among the spherical space forms (see [63], [33] and the more recent [55]). However, at present no conclusive results that either rule out non simply connected topologies or identifies a particular preferred candidate are yet available.

The results of [46] analyze the problem of cosmic topology from the point of view of the noncommutative geometry model. Namely, assuming that one takes as the action functional for gravity not the usual Einstein-Hilbert action, but the modified gravity model given by the (nonperturbative) spectral action, then one can investigate whether the explicit form of this action functional, computed for the more likely candidates for non simply connected cosmic topologies will deliver results that agree with the ordinary simply connected case or not. If they do agree, then the spectral action functional, like the usual Einstein-Hilbert action of general relativity, does not detect different topologies, while if one has different predictions for different topologies, in terms of cosmological effects that can possibly be detected in observational data, then one may have a theoretical reason for a preferred choice of a nontrivial cosmic topology, or a reason to rule out all the available candidates.

The results obtained in [46] fall in an intermediate range. Namely, one finds that the cosmic topology candidates that are spherical space forms (including the simply connected case of the sphere $S^{3}$ itself) give rise to a slow-roll inflation potential of a certain form, while the flat candidates like 
3-dimensional flat tori give rise to a different form of inflation potential. We discuss briefly at the end of this section, after recalling more in detail the results of [46], some ongoing work, aimed at improving the results of [46] with the additional contribution of the matter terms coming from the noncommutative space $F$, and possibly distinguish the different topologies, not only the underlying spherical and flat geometries.

4.3. Cosmology and the Poisson summation formula. We concentrated in [46] on the three examples among the candidate cosmic topology, which are regarded as being the most promising: the quaternionic cosmology, which is the quotient of $S^{3}=S U(2)$ by the finite group of quaternion units, the Poincaré homology 3-sphere, which is the quotient of $S^{3}$ by the binary icosahedral group, and the flat tori. A more extensive treatment covering all the other spherical forms is going to appear in a paper in preparation by Kevin Teh.

The method for computing the nonperturbative form of the spectral action in these cases is the one used in the recent paper of Chamseddine and Connes [20] in the case of the sphere $S^{3}$, and it is based on the Poisson summation formula

$$
\sum_{n \in \mathbb{Z}} h(x+\lambda n)=\frac{1}{\lambda} \sum_{n \in \mathbb{Z}} \exp \left(\frac{2 \pi i n x}{\lambda}\right) \widehat{h}\left(\frac{n}{\lambda}\right)
$$

for $\lambda \in \mathbb{R}_{+}^{*}$ and $x \in \mathbb{R}$, with

$$
\widehat{h}(x)=\int_{\mathbb{R}} h(u) e^{-2 \pi i u x} d u .
$$

The main idea on how to apply this technique to the computation of the spectral action is the following. One needs to know explicitly the spectrum of the Dirac operator, which can be computed in the case of the spherical space forms from the spectrum on the 3-sphere, using the generating functions technique of [3] to compute the correct multiplicities. It is also known for the flat tori. One then writes the expression for $\operatorname{Tr}(f(D / \Lambda))$ as a finite number of sums over lattices, by separating the spectrum of $D$ into a union of arithmetic progressions $\lambda_{n, i}$, parameterized by the integers $n \in \mathbb{Z}$. One also needs to be able to rewrite the multiplicities computed with the method of [3] as polynomial functions $m_{\lambda_{n, i}}=P_{i}\left(\lambda_{n, i}\right)$ evaluated at the corresponding eigenvalue. This step is computationally challenging, but it can be obtained with the help of computer calculations with Mathematica. One can then write the nonperturbative spectral action in the form

$$
\operatorname{Tr}(f(D / \Lambda))=\sum_{i} \sum_{n \in \mathbb{Z}} P_{i}\left(\lambda_{n, i}\right) f\left(\lambda_{n, i} / \Lambda\right)
$$

In the case of the standard simply connected case of the spherical topology $S^{3}$ with the uniform metric, the calculation of the nonperturbative spectral action was obtained in [20]. The Dirac spectrum is $\pm a^{-1}\left(\frac{1}{2}+n\right)$ for $n \in \mathbb{Z}$, with multiplicity $n(n+1)$, so that one has

$$
\operatorname{Tr}(f(D / \Lambda))=(\Lambda a)^{3} \widehat{f}^{(2)}(0)-\frac{1}{4}(\Lambda a) \widehat{f}(0)+O\left((\Lambda a)^{-k}\right)
$$

for any $k>0$, with $\widehat{f}^{(2)}$ the Fourier transform of $v^{2} f(v)$. One can then pass to 4-dimensions by choosing a compactification of a Euclidean rotation of the 4-dimensional Minkowskian spacetime. It is natural to compactify the topological cylinder on the spatial 3-manifold to a product with a circle of some size $\beta$. Thus, in the case of the 3 -sphere, one is looking at the 4-dimensional Riemannian manifold $S^{3} \times S^{1}$, for which the same method then gives the spectral action of the form

$$
\operatorname{Tr}\left(h\left(D^{2} / \Lambda^{2}\right)\right)=\pi \Lambda^{4} a^{3} \beta \int_{0}^{\infty} u h(u) d u-\frac{1}{2} \pi \Lambda a \beta \int_{0}^{\infty} h(u) d u+O\left(\Lambda^{-k}\right),
$$

where

$$
\begin{aligned}
& g(u, v)=2 P(u) h\left(u^{2}(\Lambda a)^{-2}+v^{2}(\Lambda \beta)^{-2}\right) \\
& \widehat{g}(n, m)=\int_{\mathbb{R}^{2}} g(u, v) e^{-2 \pi i(x u+y v)} d u d v
\end{aligned}
$$


4.4. A coupling of geometry and inflation. As observed already in [20], perturbations of the Dirac operator of the form $D^{2} \mapsto D^{2}+\phi^{2}$ give rise, in the nonperturbative calculation of the spectral action, to a potential $V(\phi)$ for a scalar field coupled to gravity. In fact, one has

$$
\begin{gathered}
\left.\operatorname{Tr}\left(h\left(\left(D^{2}+\phi^{2}\right) / \Lambda^{2}\right)\right)\right)=\pi \Lambda^{4} \beta a^{3} \int_{0}^{\infty} u h(u) d u-\frac{\pi}{2} \Lambda^{2} \beta a \int_{0}^{\infty} h(u) d u \\
+\pi \Lambda^{4} \beta a^{3} \mathcal{V}\left(\phi^{2} / \Lambda^{2}\right)+\frac{1}{2} \Lambda^{2} \beta a \mathcal{W}\left(\phi^{2} / \Lambda^{2}\right)
\end{gathered}
$$

where the potential is given by terms of the form

$$
\mathcal{V}(x)=\int_{0}^{\infty} u(h(u+x)-h(u)) d u, \quad \mathcal{W}(x)=\int_{0}^{x} h(u) d u .
$$

What is especially interesting in this type of potential, as shown in [46] is the fact that they give rise to a slow-roll inflation model where the scalar field $\phi$ determines the inflation. In fact, one first observes that the potential obtained from the computation of the spectral action for the Riemannian 4-dimensional manifold still makes sense when one rotates back to the Minkowskian case, so that one can consider again a Friedmann metric on $S^{3} \times \mathbb{R}$, or more generally on a 4-manifold $M=S \times \mathbb{R}$, where $S$ is one of the other 3-dimensional space forms we discuss below. One can then compute the slow-roll parameters (3.4) and (3.5) for the potential $V(\phi)$ and the resulting spectral index and tensor to scalar ratio.

One finds expressions of the form

$$
\begin{gathered}
\epsilon(x)=\frac{m_{P l}^{2}}{16 \pi}\left(\frac{h(x)-2 \pi(\Lambda a)^{2} \int_{x}^{\infty} h(u) d u}{\int_{0}^{x} h(u) d u+2 \pi(\Lambda a)^{2} \int_{0}^{\infty} u(h(u+x)-h(u)) d u}\right)^{2} \\
\eta(x)=\frac{m_{P l}^{2}}{8 \pi} \frac{h^{\prime}(x)+2 \pi(\Lambda a)^{2} h(x)}{\int_{0}^{x} h(u) d u+2 \pi(\Lambda a)^{2} \int_{0}^{\infty} u(h(u+x)-h(u)) d u} \\
-\frac{m_{P l}^{2}}{16 \pi}\left(\frac{h(x)-2 \pi(\Lambda a)^{2} \int_{x}^{\infty} h(u) d u}{\int_{0}^{x} h(u) d u+2 \pi(\Lambda a)^{2} \int_{0}^{\infty} u(h(u+x)-h(u)) d u}\right)^{2},
\end{gathered}
$$

where in the case of a Lorentzian Friedmann metric one has an inverse relation between the energy scale $\Lambda$ and the expansion factor $a$, with $\Lambda(t) \sim 1 / a(t)$, which one does not have in the Euclidean model. The expressions are also (as they should be) independent of the spurious parameter $\beta$ introduced in the Euclidean compactification used for the explicit computation of the spectral action, which only enters as an overall multiplicative factor in the potential $V(\phi)$, hence it drops out of the expression for the slow-roll coefficients.

The first nontrivial case computed in [46] is the quaternionic cosmology, given by the quotient $S=S U(2) / Q 8$ by the finite group of quaternion units $\left\{ \pm 1, \pm \sigma_{k}\right\}$. The Dirac spectrum for this 3 -manifold is known explicitly from a calculation of [34]: it is given by

$$
\begin{aligned}
& \frac{3}{2}+4 k \text { with multiplicity } 2(k+1)(2 k+1) \\
& \frac{3}{2}+4 k+2 \text { with multiplicity } 4 k(k+1) .
\end{aligned}
$$

This determines polynomial interpolation functions for the spectral multiplicities of the form

The spectral action is then given by

$$
\begin{aligned}
& P_{1}(u)=\frac{1}{4} u^{2}+\frac{3}{4} u+\frac{5}{16} \\
& P_{2}(u)=\frac{1}{4} u^{2}-\frac{3}{4} u-\frac{7}{16} .
\end{aligned}
$$

$$
\operatorname{Tr}(f(D / \Lambda))=\frac{1}{8}(\Lambda a)^{3} \widehat{f}^{(2)}(0)-\frac{1}{32}(\Lambda a) \widehat{f}(0)+O\left(\Lambda^{-k}\right)
$$


for all $k>0$, which is the same as $1 / 8$ of the spectral action for $S^{3}$. Setting $g_{i}(u)=P_{i}(u) f(u / \Lambda)$, one then obtains for the 4-dimensional case

$$
\operatorname{Tr}(f(D / \Lambda))=\frac{1}{4}\left(\widehat{g}_{1}(0)+\widehat{g}_{2}(0)\right)+O\left(\Lambda^{-k}\right),
$$

from Poisson summation formula. One sees then explicitly that this case gives the same slow-roll parameters as the 3 -sphere.

It is interesting to observe that in this case, as in the other ones discussed below, although the 3-manifold admits different inequivalent spin structures, for which the Dirac operator has genuinely different spectrum, when the nonperturbative spectral action is computed with the method described above for the different spin structures one obtains the same spectral action.

The second example computed in [46] is the dodecahedral cosmology, that is, the Poincaré homology 3 -sphere, obtained as the quotient $S^{3} / \Gamma$ by the binary icosahedral group of 120 elements, which is the most extensively studied candidate for a non-trivial cosmic topology, [14].

In this case the spectrum is computed from the Dirac eigenvalues of $S^{3}$ with different multiplicities obtained by computing explicitly the generating functions of [3] using the explicit form of the 120 group elements. The generating functions for the spectral multiplicities are then of the form

$$
F_{+}(z)=\sum_{k=0}^{\infty} m\left(\frac{3}{2}+k, D\right) z^{k} \quad \text { and } \quad F_{-}(z)=\sum_{k=0}^{\infty} m\left(-\left(\frac{3}{2}+k\right), D\right) z^{k},
$$

and are computed in closed form in [46] as ratios of completely explicit (though compicated) polynomials in $z$. These in turn then determine the polynomial interpolation functions for the spectral multiplicities as a set of 60 explicit polynomials $P_{i}(u)$ with the property that

$$
\sum_{j=0}^{59} P_{j}(u)=\frac{1}{2} u^{2}-\frac{1}{8}
$$

This again can be used to explicitly compute the spectral action. In terms of functions $g_{j}(u)=$ $P_{j}(u) f(u / \Lambda)$, one finds

$$
\begin{aligned}
& \operatorname{Tr}(f(D / \Lambda))=\frac{1}{60} \sum_{j=0}^{59} \widehat{g}_{j}(0)+O\left(\Lambda^{-k}\right) \\
& =\frac{1}{60} \int_{\mathbb{R}} \sum_{j} P_{j}(u) f(u / \Lambda) d u+O\left(\Lambda^{-k}\right)
\end{aligned}
$$

using again the Poisson summation formula. The result is exactly $1 / 120$ of the spectral action for $S^{3}$, so that again one obtains the same slow-roll parameters as in the sphere case.

If one looks instead at the example of the flat tori, one finds a genuinely different form of the slow-roll potential and parameters. In the case of the flat tori again the Dirac spectrum is known to be of the form

$$
\pm 2 \pi\left\|(m, n, p)+\left(m_{0}, n_{0}, p_{0}\right)\right\|,
$$

form $(m, n, p) \in \mathbb{Z}^{3}$ with multiplicity 1 and a constant vector $\left(m_{0}, n_{0}, p_{0}\right)$, which depends on the spin structure. The spectral action is then given by

$$
\operatorname{Tr}\left(f\left(D_{3}^{2} / \Lambda^{2}\right)\right)=\sum_{(m, n, p) \in \mathbb{Z}^{3}} 2 f\left(\frac{4 \pi^{2}\left(\left(m+m_{0}\right)^{2}+\left(n+n_{0}\right)^{2}+\left(p+p_{0}\right)^{2}\right)}{\Lambda^{2}}\right),
$$

as one computes by applying the Poisson summation formula

$$
\sum_{\mathbb{Z}^{3}} g(m, n, p)=\sum_{\mathbb{Z}^{3}} \widehat{g}(m, n, p)
$$

with

$$
\widehat{g}(m, n, p)=\int_{\mathbb{R}^{3}} g(u, v, w) e^{-2 \pi i(m u+n v+p w)} d u d v d w
$$




$$
g(m, n, p)=f\left(\frac{4 \pi^{2}\left(\left(m+m_{0}\right)^{2}+\left(n+n_{0}\right)^{2}+\left(p+p_{0}\right)^{2}\right)}{\Lambda^{2}}\right) .
$$

Thus, the spectral action for the flat tori is given by

$$
\operatorname{Tr}\left(f\left(D_{3}^{2} / \Lambda^{2}\right)\right)=\frac{\Lambda^{3}}{4 \pi^{3}} \int_{\mathbb{R}^{3}} f\left(u^{2}+v^{2}+w^{2}\right) d u d v d w+O\left(\Lambda^{-k}\right)
$$

and for the 4-dimensional $X=T^{3} \times S_{\beta}^{1}$ one gets

$$
\operatorname{Tr}\left(h\left(D_{X}^{2} / \Lambda^{2}\right)\right)=\frac{\Lambda^{4} \beta \ell^{3}}{4 \pi} \int_{0}^{\infty} u h(u) d u+O\left(\Lambda^{-k}\right),
$$

using

$$
\begin{gathered}
\sum_{(m, n, p, r) \in \mathbb{Z}^{4}} 2 h\left(\frac{4 \pi^{2}}{(\Lambda \ell)^{2}}\left(\left(m+m_{0}\right)^{2}+\left(n+n_{0}\right)^{2}+\left(p+p_{0}\right)^{2}\right)+\frac{1}{(\Lambda \beta)^{2}}\left(r+\frac{1}{2}\right)^{2}\right), \\
g(u, v, w, y)=2 h\left(\frac{4 \pi^{2}}{\Lambda^{2}}\left(u^{2}+v^{2}+w^{2}\right)+\frac{y^{2}}{(\Lambda \beta)^{2}}\right), \\
\sum_{(m, n, p, r) \in \mathbb{Z}^{4}} g\left(m+m_{0}, n+n_{0}, p+p_{0}, r+\frac{1}{2}\right)=\sum_{(m, n, p, r) \in \mathbb{Z}^{4}}(-1)^{r} \widehat{g}(m, n, p, r) .
\end{gathered}
$$

Introducing the perturbation $D^{2} \mapsto D^{2}+\phi^{2}$ one then finds

$$
\operatorname{Tr}\left(h\left(\left(D_{X}^{2}+\phi^{2}\right) / \Lambda^{2}\right)\right)=\operatorname{Tr}\left(h\left(D_{X}^{2} / \Lambda^{2}\right)\right)+\frac{\Lambda^{4} \beta \ell^{3}}{4 \pi} \mathcal{V}\left(\phi^{2} / \Lambda^{2}\right)
$$

which gives as slow-roll potential

where we have

$$
V(\phi)=\frac{\Lambda^{4} \beta \ell^{3}}{4 \pi} \mathcal{V}\left(\phi^{2} / \Lambda^{2}\right)
$$

$$
\mathcal{V}(x)=\int_{0}^{\infty} u(h(u+x)-h(u)) d u .
$$

This shows that the slow-roll parameters are now genuinely different from the spherical cases. One has

$$
\begin{gathered}
\epsilon=\frac{m_{P l}^{2}}{16 \pi}\left(\frac{\int_{x}^{\infty} h(u) d u}{\int_{0}^{\infty} u(h(u+x)-h(u)) d u}\right)^{2} \\
\eta=\frac{m_{P l}^{2}}{8 \pi}\left(\frac{h(x)}{\int_{0}^{\infty} u(h(u+x)-h(u)) d u}-\frac{1}{2}\left(\frac{\int_{x}^{\infty} h(u) d u}{\int_{0}^{\infty} u(h(u+x)-h(u)) d u}\right)^{2}\right) .
\end{gathered}
$$

4.5. A coupling of topology and inflation? The results of [46] reveal the unexpected fact that, in the NCG model of gravity based on the spectral action functional, the possible slow-roll potentials that can arise as nonperturbative effects in the spectral action are strongly constrained by the underlying spatial geometry of the universe.

However, in [46] one works under the approximation of considering the spectral action on the manifold $M$ itself and not on the product space $M \times F$. In case of a nontrivial spatial topology, one can in fact consider more refined models, where the product $M \times F$ is replaced by a nontrivial fibration with noncommutative fibers. This affects the asymptotic expansion of the spectral action by introducing additional fields, as a recent computation by Ćaćić shows.

Thus, it is reasonable to expect that, if the same type of nonperturbative calculation of the spectral action is carried out not on $M$ itself, but on a nontrivial fibration on $M$ with fiber $F$, one may be able to obtain some more refined constraints on the type of inflation potentials allowed in the model, and show in particular that different spherical forms will give rise to different inflation potentials. This would improve the coupling of geometry and inflation observed in the model to a much more 
interesting couping of topology and inflation, which may be able to rule out, within the model, certain candidate cosmic topologies and select others. This is part of ongoing work.

\section{Questions And PeRspectives}

I will sketch here a brief outline of some of the directions in which this research project is currently evolving and what further results one expects to be able to obtain with the methods described above.

5.1. Dark matter and dark energy. The model of [21] has three active and three sterile neutrinos as in the $\nu$ MSM model, see [39], [59], [60], though in [21], unlike in the $\nu$ MSM model, it is assumed that the three sterile neutrinos all have masses well above the electroweak scale. The see-saw relation $Y_{\nu}^{T} M^{-1} Y_{\nu}$ for neutrino masses is obtained in [21] geometrically from the restriction of the Dirac operator $D$ to the subspace of $\mathcal{H}_{F}$ which corresponds to the neutrinos sector, see Lemma 1.225 of [27].

Thus, in terms of developing dark matter models within this framework, the first natural candidate would be models based on right handed neutrinos with Majorana mass terms, see [59], [60] and the sterile neutrinos scenarios of [39].

Cosmology has already proven to put tight constraints on both active and sterile neutrinos and to their possible interactions. In the particular case of models based on Majorana mass terms for right handed neutrinos, a discussion of the current status of exclusion curves based on cosmological data is given in [39]. This type of constraints can be used to obtain further exclusion curves on the boundary conditions at unification for our NCG model and on the free parameters $f_{2}$ and $f_{4}$.

In fact, for the right handed neutrinos with Majorana masses to give rise to plausible dark matter models as in Shaposhnikov-Tkachev [60], Shaposhnikov [59], and Kusenko [39], one needs at least one (or more) of the sterile neutrino Majorana masses to be below the electroweak scale. In the detailed discussion given in [39] one sees that, for example, one could have two of the three Majorana masses that remain very large, well above the electroweak scale, possibly close to unification scale, while a third one lowers below the electroweak scale, so that the very large Majorana masses still account for the see-saw mechanism, while the smaller one provides a candidate dark matter particle.

The default boundary conditions of [1] used in the RGE analysis of [45] do not allow for any of the Majorana masses to descend below the electroweak scale. However, using the broader search for variable boundary conditions provided in the work [38], it will be possible to look for regions in the manifold of boundary conditions at unification scale with the desired property that at least one of the Majorana masses reaches, in the more modern universe, a scale at which it becomes feasible as a dark matter model in the sense of [60], [39]. This will impose exclusion regions on the initial conditions according to the matching of the resulting dark matter scenarios to cosmological data.

In addition to these possible dark matter scenarios based on Majorana masses for right handed neutrinos, the new version of the NCG model that incorporates supersymmetry as developed in [9] makes it possible to implement other dark matter models based on the available spectrum of supersymmetric particles. A more detailed strategy in this case will require first a better understanding of how the current supersymmetric model developed in [9] can be extended to the full MSSM. Then the general strategy will again be based on renormalization group analysis as in [45], extended to accommodate the larger particle content, non-perturbative effects at low energies, also to be recalculated in the modified finite geometry needed for supersymmetric models, and exclusion curves on the initial condition of the RGE at unification that fit cosmological tests on the resulting dark matter models based on supersymmetric particles instead of neutrinos.

The presence in the NCG model of a variable effective cosmological constant seems promising in terms of deriving dark energy models. In order to be able to do that, one needs to control the nonperturbative effects on this running at low energies sufficient to reach the epoch of the universe where dark energy models become relevant.

In addition to the variable effective cosmological constant, another possible source of dark energy models is the dilaton field. The present model includes a dilaton field, which comes from making dynamical the energy cutoff in the spectral action, as shown in [18]. The dilaton field was already used 
in [18] to recover a version of the Randall-Sundrum model, where instead of having brane geometry and extra dimensions, the finite noncommutative space plays the role of a "two point space" which produces a similar effect to the pair of D-branes in the Randall-Sundrum scenario, $c f$. [42], and it also has direct relevance to inflation models. This can have relevance also for models of dark energy based on NCG. In fact, the dilaton field is recognized as one of the possible sources of dark energy models. More precisely, as the cutoff in the ordinary spectral action appears in all the gravitational terms in the asymptotic expansion of the spectral action, which give the Einstein-Hilbert and gravitational terms, in the case of the spectral action with dilaton field one finds in the asymptotic expansion a dilaton potential, which can contribute a repulsive force like the cosmological constant. One can use this to develop a scenario where dark energy is modeled by the dilaton field so that, when cosmic time goes to infinity and the dilaton field goes to zero, the potential recovers the cosmological constant.

5.2. Bariogenesis via leptogenesis. Another question that is central to modern cosmology is the apparent matter/antimatter asymmetry in the universe. In order to test our available mathematical model for the capacity to make predictions of direct physical interest, one should definitely try to understand what such a model can say about the issue of a primordial matter/antimatter asymmetry. This problem is in fact strictly related to the question discussed above of the dark matter problem.

In a famous early work on the subject, Sakharov outlined three necessary conditions a particle physics model must possess in order to be able to account for matter/antimatter asymmetry. The first criterion is the violation of the baryon number symmetry, the second is CP-violation, and the third is departure from thermal equilibrium (as is the case in an expanding universe). The main focus in approaching this problem has then been on mechanisms that produce baryon number asymmetry (baryogenesis). In particular, since the discovery of neutrino oscillations which imposed the extension of the MSM to include right handed neurinos and neutrino masses, physicists concentrated especially on mechanisms for baryon asymmetry that are guided by neutrino physics (leptogenesis). For some recent detailed accounts of the baryogenesis and leptogenesis problems see [22] and also [10], [61].

In the context of the $\nu \mathrm{MSM}$, the question of baryogenesis via leptogenesis has been investigated in [2], [40], [58], including its implications on the dark matter question. It should be mentioned here, because it will be useful below, that in the $\nu \mathrm{MSM}$ the leptogenesis is completely guided by the extra degrees of freedom coming from the right handed neutrinos, while the NCG model appears to have a broader spectrum of possibilities for baryogenesis, which are not present in the $\nu$ MSM.

First, one needs to verify that the NCG model satisfies the Sakharov conditions. As in the $\nu \mathrm{MSM}$, the model contains a CP-violating phase in the lepton sector and a CKM matrix CP-violating phase in the quark sector. The lepton number is violated by the Majorana neutrino masses. In the $\nu \mathrm{MSM}$ one argues that the $B+L$ symmetry is broken by the electroweak anomaly. A study of anomalies within the NCG setting is presented in $\S 1$ of [27]. The result is a computation of anomalies in noncommutative geometry based on certain cyclic cocycles related to the index cocycle of Connes-Moscovici [28]. One may be able to use similar anomaly computation techniques in NCG to check the $B+L$ violation criterion in the NCG model. Moreover, the third Sakharov condition may be in fact easier to check in the NCG model than in the $\nu$ MSM model. In fact, the $\nu$ MSM model is limited by the fact that it does not have a scale for the Higgs mass hence an electroweak transition, so that the so called "electroweak baryogenesis" cannot take place within the $\nu$ MSM model. These however are included in the NCG model, which at the same time also has the same mechanism present in the $\nu \mathrm{MSM}$ for out of thermal equilibrium states coming from the size of the Yukawa coupling of the sterile neutrinos to the other fermions. This second mechanism is in turn more difficult to implement in the NCG model than in the $\nu$ MSM. This appears to be closely related to the presence of a dilaton field in the noncommutative geometry model, as shown in [18].

5.3. High energy regime and noncommutative spacetime. Certain models of string theory compactifications predict the appearance of noncommutativity in the spacetime coordinates, replacing the ordinary $\mathbb{R}^{4}$ geometry of flat spacetime by a Moyal type noncommutative $\mathbb{R}_{\theta}^{4}$. An extensive 
investigation of quantum field theory on these Moyal noncommutative spaces, and of possible signatures of such noncommutativity in cosmological data have been carried out in recent years. In fact, this hypothesis of noncommutativity also has cosmological implications, which have been investigated, for example in [48] and essentially ruled out by comparison with observational results in cosmology.

That type of model based on noncommutative deformations of the underlying spacetime coordinates is, at present, essentially unrelated to the noncommutative geometry models of particle physics we have been considering here, where no noncommutativity arises in the spacetime coordinates, and only the extra dimensions are given by a noncommutative space. In particular, the existing work on possible cosmological signatures of noncommutativity, such as [48], which are carried out to test the Moyal type deformations of spacetime coordinates, do not apply to our models.

However, speculations exist to the effect that, when going to sufficiently high energies (above unification scale and moving towards the Planck scale) the amount of noncommutativity in the models we are looking at might increase. This would mean that, instead of dealing with a product geometry $X=M \times F$, where $M$ is an ordinary manifold and $F$ a noncommutative space, the manifold $M$ itself may undergo a phase transition to a noncommutative geometry, perhaps of the same Moyal nature as those obtained in string theory compactifications, or perhaps in some other, more elaborate, form. There is at present little evidence within the structure of the mathematical model to support such a conjecture. However, it is a hypothesis worth investigating, since it would have relevance both in understanding if the model can avoid a singularity at high energy and also in establishing a bridge to the existing results for Moyal deformations and the existing estimates of possible cosmological signatures of that, very different, type of noncommutativity.

Since the noncommutativity of spacetime coordinates, within the type of models we are considering, can appear only at an energy scale above unification, in cosmological terms this would affect the universe at an epoch which is even more remote than that considered in our early universe models that live between the unification and the electroweak era. It then becomes more challenging to see what possible cosmological signatures may remain detectable in the modern universe. The investigations that ruled out spacetime noncommutativity again would not apply to test its possible presence only in that primordial phase of the universe, hence a different strategy would be required to test this hypothesis.

5.4. Possible relations to string theory models. The question above suggests a longer term direction of investigation, which is the possible relation of the $K$-theoretically six dimensional noncommutative geometry $F$ (in a version involving supersymmetry) to low energy limits of Calabi-Yau compactifications of string theory. The fact that noncommutative spaces may arise as low energy limits of commutative geometries is not new: a similar idea was used for example in [32]. A preliminary step for this type of analysis is a thorough classification of all the possible finite geometries and their properties, extending the recent analysis given in [19]. This was carried out in [13]. Another preliminary step towards relating the NCG model to string theory vacua would be investigating the possibilities for noncommutative Calabi-Yau geometries. A good notion exists of Calabi-Yau algebras (and Calabi-Yau categories) generalizing the usual commutative Calabi-Yau manifolds. This has the advantage that it can be expressed in terms of conditions depending on properties of the cyclic cohomology, and on the presence of supersymmetry, [8], [29], [35]. One can first investigate the question of the existence of finite noncommutative geometries that satisfy a similar Calabi-Yau condition and relate these to the finite geometries of the NCG models. A good possible point of approach would be the notion of Calabi-Yau over finite fields considered in [15] which already provides examples of (commutative) finite geometries satisfying a Calabi-Yau condition, which one can then try to "thicken" to noncommutative spaces just as it happens with the "two-point space" of the NCG model of particle physics.

5.5. Kasner metrics and mixmaster cosmologies. In [45] we showed how the running of the effective gravitational constant affects the solutions of the Friedmann equation and the propagation of gravitational waves, showing the presence of amplification phenomena. The generation and behavior 
of the gravitational waves is closely linked, in theoretical cosmology, to the value of the spectral index which we have also analyzed in the model. So another relevant question to investigate is how to relate directly the behavior of gravitational waves to the information we obtain in the model on the slow roll parameter. Aiming at a more detailed study of how the behavior of gravitational waves is affected in the NCG models, it may be useful to consider models of non-isotropic cosmologies. In fact, it was shown in [51] that, in the case of the Kasner metrics, the form of the gravitational waves equation itself changes due to the presence of the non-commutative space $F$, and not only through the different behavior of the effective gravitational constant in the model. Thus, applying the analysis of [45] to these well known models of non-isotropic cosmologies may help in gaining a more detailed understanding of how the generation and propagation of gravitational waves is affected and how this may be related to the slow roll parameters.

Moreover, the example of Kasner metrics is interesting to look at in this context also for its relation to mixmaster cosmological models. Unlike the standard cosmological models based on highly symmetric solutions of the Einstein equations, the mixmaster universe models first introduced in the early 1970s (see [7] and also [5]) present a very interesting example of cosmological models with strong anisotropies and with a chaotic evolution [37] consisting of successive Kasner eras during which the universe is modeled by an anisotropic Kasner metric. The relevance today of mixmaster cosmological models is mostly in possible scenarios of pre-inflationary evolution and in certain approaches to quantum gravity (see [50]).

The dynamics of mixmaster cosmology can be modeled by a discrete dynamical system based on the Gauss shift of the continued fraction expansion, [37], [47]. In fact, it was shown more precisely in [43] and [44] that the mixmaster solutions are parameterized by geodesics on the modular curve $X_{0}(2)$. It was shown in [44] that these classes of solutions, which are specified by geometric properties of geodesics on the modular curve (e.g. how far they wander into the cusps), have a moduli space which is in fact a noncommutative space given by a Cuntz-Krieger $C^{*}$-algebra. In [43] it was also shown how the mixmaster cosmology relates to the theory of noncommutative boundaries of modular curves, to the Gauss-Kuzmin operator (the transfer operator of the shift of the continued fraction expansions for finite index subgroups of the modular group) and through that to the Selberg zeta function.

Mixmaster cosmologies nowadays have relevance to models of chaotic inflation and brane-world scenarios. Thus, investigating how our NCG early universe model behaves in the case of such cosmologies may further improve our understanding of inflationary mechanisms available within the NCG framework and will connect the NCG models under investigation to the mathematical formulation in terms of algebraic and noncommutative spaces derived in [43] and [44]. 


\section{REFERENCES}

[1] S. Antusch, J. Kersten, M. Lindner, M. Ratz, M.A. Schmidt Running neutrino mass parameters in see-saw scenarios, JHEP 03 (2005) 024, hep-ph/0501272v3.

[2] T. Akasa, M. Laine, M. Shaposhnikov, On the hadronic contribution to sterile neutrino production, Journal of High Energy Physics (2006) 053, 28pp.

[3] C. Bär, The Dirac operator on space forms of positive curvature. J. Math. Soc. Japan 48 (1996), no. 1, 69-83.

[4] J.W. Barrett, A Lorentzian version of the non-commutative geometry of the standard model of particle physics, J.Math.Phys. 48 (2007) 012303.

[5] J. D. Barrow. Chaotic behaviour and the Einstein equations, in "Classical General Relativity", pp.25-41, Cambridge Univ. Press, 1984.

[6] J.D. Barrow, Gravitational memory? Phys. Rev. D Vol.46 (1992) N.8 R3227, 4pp.

[7] V.A. Belinskii, E.M. Lifshitz, I.M. Khalatnikov, Oscillatory approach to the singular point in relativistic cosmology, Soviet Physics Uspekhi, Vol.13 (1971) N.6, 745-765.

[8] D. Berenstein, R.G. Leigh, Non-commutative Calabi-Yau manifolds, Physics Letters B, Vol.499, N.1 (2001) 207214.

[9] T. van den Broek, W.D. van Suijlekom, Supersymmetric QCD and noncommutative geometry, arXiv:1003.3788.

[10] W. Buchmuller, R.D. Peccei, T. Yanagida, Leptogenesis as the origin of matter, Ann.Rev.Nucl.Part.Sci. 55 (2005) 311-355.

[11] M. Buck, M. Fairbairn, M. Sakellariadou, Inflation in models with conformally coupled scalar fields: an application to the noncommutative spectral action, arXiv:1005.1188.

[12] E.V. Bugaev, V.B. Petkov, A.N. Gaponenko, P.A. Klimai, M.V. Andreev, A.B. Chernyaev, I.M. Dzaparova, D.D. Dzhappuev, Zh.Sh. Guliev, N.S. Khaerdinov, N.F. Klimenko, A.U. Kudzhaev, A.V. Sergeev, V.I. Volchenko, G.V. Volchenko, A.F. Yanin, Experimental search of bursts of gamma rays from primordial black holes using different evaporation models, arXiv:0906.3182.

[13] B. Cacic, Moduli spaces of Dirac operators for finite spectral triples, arXiv:0902.2068.

[14] S. Caillerie, M. Lachièze-Rey, J.P. Luminet, R. Lehoucq, A. Riazuelo, J. Weeks, A new analysis of the Poincaré dodecahedral space model, arXiv:0705.0217.

[15] P. Candelas, X. de la Ossa, F. Rodriguez-Villegas, Calabi-Yau manifolds over finite fields, I. hep-th/0012233.

[16] A. Chamseddine, A. Connes, Universal formulas for noncommutative geometry actions, Phys. Rev. Letters, Vol.77, N.24, (1996) 4868-4871.

[17] A. Chamseddine, A. Connes, The spectral action principle, Commun. Math. Phys. 186 (1997) 731-750.

[18] A. Chamseddine, A. Connes, Scale invariance in the spectral action, J.Math.Phys. 47 (2006) 063504.

[19] A. Chamseddine, A. Connes, Conceptual explanation for the algebra in the noncommutative approach to the Standard Model, (previously circulated with title "A dress for SM the beggar"), Phys. Rev. Lett. 99, 191601 (2007), 4pp.

[20] A. Chamseddine, A. Connes, The uncanny precision of the spectral action, arXiv:0812.0165.

[21] A. Chamseddine, A. Connes, M. Marcolli, Gravity and the standard model with neutrino mixing, Advances in Theoretical and Mathematical Physics, 11 (2007) 991-1090.

[22] M.C. Chen, TASI 2006 Lectures on Leptogenesis, hep-ph/0703087.

[23] A. Connes, Noncommutative geometry. Academic Press, 1994.

[24] A. Connes, Geometry from the spectral point of view. Lett. Math. Phys. 34 (1995), no. 3, 203-238.

[25] A. Connes, Gravity coupled with matter and foundation of noncommutative geometry. Commun. Math. Phys., 182 (1996) 155-176.

[26] A. Connes, Noncommutative Geometry and the standard model with neutrino mixing, JHEP 0611 (2006) 081.

[27] A. Connes, M. Marcolli, Noncommutative geometry, quantum fields and motives. Colloquium Publications, Vol.55, American Mathematical Society, 2008.

[28] A. Connes, H. Moscovici, The local index formula in noncommutative geometry. Geom. Funct. Anal. 5 (1995), no. $2,174-243$

[29] K. Costello, Topological conformal field theories and Calabi-Yau categories, Adv. Math., Vol.210 (2007) N.1, 165214.

[30] A. De Simone, M.P. Hertzberg, F. Wilczek, Running Inflation in the Standard Model, Phys. Lett. B 678: 1-8 (2009).

[31] J.F. Donoghue, General relativity as an effective field theory: The leading quantum corrections, Phys. Rev. D50 (1994) 3874-3888.

[32] J. Fröhlich, K. Gawedzki, Conformal field theory and geometry of strings. Mathematical quantum theory. I. Field theory and many-body theory (Vancouver, BC, 1993), 57-97, CRM Proc. Lecture Notes, 7, Amer. Math. Soc., Providence, RI, 1994.

[33] E. Gausmann, R. Lehoucq, J.P. Luminet, J.P. Uzan, J. Weeks, Topological lensing in spherical spaces, Class. Quantum Grav. 18 (2001) 5155-5186.

[34] N. Ginoux, The spectrum of the Dirac operator on $\mathrm{SU}_{2} / \mathrm{Q}_{8}$. Manuscripta Math. 125 (2008), no. 3, 383-409. 
[35] V. Ginzburg, Calabi-Yau algebras, arXiv:math/0612139.

[36] A.H. Guth, Phase transitions in the very early universe, in "The very early universe" (G.W.Gibbons, S.W.Hawking, S.T.C.Siklos, Eds.) pp. 171-204, Cambridge University Press, 1982.

[37] I. M. Khalatnikov, E.M. Lifshitz, K.M. Khanin, L.N. Shchur, Ya.G. Sinai, On the stochasticity in relativistic cosmology, Journal of Statistical Physics, Vol.38, N.1-2 (1985) 97-114.

[38] D. Kolodrubetz, M. Marcolli, Boundary conditions of the RGE flow in noncommutative cosmology, arXiv:1006.4000.

[39] A. Kusenko, Sterile neutrinos: the dark side of the light fermions, arXiv:0906.2968.

[40] M. Laine, M. Shaposhnikov, Sterile neutrino dark matter as a consequence of $\nu M S M$-induced lepton asymmetry, Journal of Cosmology and Astroparticle Physics,

[41] A.D. Linde, Gauge theories, time-dependence of the gravitational constant and antigravity in the early universe, Phys. Letters B, Vol.93 (1980) N.4, 394-396.

[42] F. Lizzi, G. Mangano, G. Miele, Another alternative to compactification: noncommutative geometry and RandallSundrum models, Mod.Phys.Lett. A16 (2001) 1-8.

[43] Yu.I. Manin, M. Marcolli, Continued fractions, modular symbols, and non-commutative geometry, Selecta Mathematica (New Ser.), Vol. 8 N.3 (2002) 475-520.

[44] M. Marcolli, Modular curves, $C^{*}$-algebras and chaotic cosmology, in "Frontiers in Number Theory, Physics, and Geometry, II" pp.361-372, Springer Verlag, 2006.

[45] M. Marcolli, E. Pierpaoli, Early Universe models from Noncommutative Geometry, arXiv:0908.3683.

[46] M. Marcolli, E. Pierpaoli, K. Teh, The spectral action and cosmic topology, arXiv:1005.2256.

[47] D. Mayer, Relaxation properties of the mixmaster universe, Phys. Lett. A 121 (1987) N.8-9, 390-394.

[48] I. Mocioiu, M. Pospelov, R. Roiban, Limits on the Non-commutativity Scale, arXiv:hep-ph/0110011.

[49] R.N. Mohapatra, P.B. Pal, Massive neutrinos in physics and astrophysics, World Scientific, 2004.

[50] G. Montani, M.V. Battisti, R. Benini, G. Imponente, Classical and quantum features of the mixmaster singularity, arXiv:0712.3008.

[51] W. Nelson, M. Sakellariadou, Cosmology and the Noncommutative approach to the Standard Model, arXiv:0812.1657.

[52] W. Nelson, M. Sakellariadou, Natural inflation mechanism in asymptotic noncommutative geometry, Phys.Lett. B 680:263-266 (2009)

[53] W. Nelson, J. Ochoa, M. Sakellariadou, Gravitational waves in the spectral action of noncommutative geometry, arXiv:1005.4276.

[54] W. Nelson, J. Ochoa, M. Sakellariadou, Constraining the Noncommutative Spectral Action via Astrophysical Observations, arXiv:1005.4279.

[55] A. Niarchou, A. Jaffe, Imprints of spherical nontrivial topologies on the cosmic microwave background, Physical Review Letters, 99 (2007) 081302, 4pp.

[56] I.D. Novikov, A.G. Polnarev, A.A. Starobinsky, Ya.B. Zeldovich, Primordial black holes, Astron. Astrophys. 80 (1979) 104-109.

[57] A. Riazuelo, S. Caillerie, M. Lachize-Rey, R. Lehoucq, J.P. Luminet, Constraining Cosmic Topology with CMB Polarization, arXiv:astro-ph/0601433.

[58] M. Shaposhnikov, The $\nu M S M$, dark matter and baryon asymmetry of the Universe, Journal of Physics: Conference Series 39 (2006) 9-11.

[59] M. Shaposhnikov, The $\nu$ MSM, leptonic asymmetries, and properties of singlet fermions, JHEP (2008) 0808:008, $56 \mathrm{pp}$.

[60] M. Shaposhnikov, I. Tkachev, The $\nu M S M$, inflation, and dark matter, Phys.Lett. B Vol.639 (2006) 414-417.

[61] M. Trodden, Electroweak baryogenesis, Rev. Mod. Phys. 71 (1999) 1463-1500.

[62] J. Weeks, J. Gundermann, Dodecahedral topology fails to explain quadrupole-octupole alignment, Class. Quantum Grav. 24 (2007) 1863-1866.

[63] J. Weeks, R. Lehoucq, J.P. Uzan, Detecting topology in a nearly flat spherical universe, Class. Quantum Grav. 20 (2003) 1529-1542.

Division of Physics, Mathematics and Astronomy, California Institute of Technology, 1200 East CaliFornia Boulevard, Pasadena, CA 91125, USA

E-mail address: matilde@caltech.edu 